TRANSACTIONS OF THE

AMERICAN MATHEMATICAL SOCIETY

Volume 184, Octobet 1973

\title{
A COMPLETELY MITOTIC NONRECURSIVE R.E. DEGREE
}

BY

\author{
RICHARD E. LADNER
}

ABSTRACT. A nonrecursive r.e. degree $d$ is constructed that has the property that every r.e. set of degree $d$ is mitotic. The degree $d$ has several other interesting properties including the property that any two r.e. sets of degree $d$ are weak truth table equivalent.

The purpose of this paper is to prove the following:

Theorem. There exists a nonrecursive r.e. degree $\mathrm{d}$ such that every r.e. set of degree $\mathrm{d}$ is mitotic.

The notion of mitotic was defined by the author in [2]. A r.e. set is mitotic if it is the disjoint union of two r.e. sets both of the same degree. It was shown [2, Theorems 3 and 4] that there is a nonmitotic r.e. set of degree $0^{\prime}$ and that for each nonrecursive r.e. set $A$ there is a nonmitotic r.e. set recursive in $A$. From these facts one might suspect that every nonrecursive r.e. degree contains a nonmitotic r.e. set. However, as we shall show, such is not the case. The degree d that we construct has several other interesting properties. A r.e. set is strongly mitotic if it is the disjoint union of two recursively separable r.e. sets of the same degree. In [2, Theorem 5$]$ it is shown that maximal sets can be mitotic yet it is quite easy to show that no maximal set can be strongly mitotic. Hence the notion of mitotic is truly weaker than the notion of strongly mitotic. The construction of the degree $d$ actually yields the stronger result.

Corollary 1. Every r.e. set of degree $\mathrm{d}$ is strongly mitotic.

The following corollary is a consequence of the fact that every r.e. set which is mitotic is also autoreducible [2, Theorem 1].

Corollary 2. Every r.e. set of degree $\mathrm{d}$ is autoreducible.

The notion of weak truth table reducibility is defined in Rogers [3, p. 158]. As a by-product of the construction we obtain:

Received by the editors March 16, 1972 and, in revised form, February 18, 1973.

AMS (MOS) subject classifications (1970). Primary 02F25, 02F30.

Key words and phrases. Recursively enumerable, degree, mitotic, strongly mitotic, weak truth table reducibility. 
Corollary 3. Any two r.e. sets of degree d are weak trutb table equivalent.

Finally the degree $d$ will have the property that $d^{\prime \prime}=0 "$. This property and Corollaries 1 and 3 are strictly speaking not corollaries of the theorem but are corollaries of the construction. Hence we shall give their proofs after the proof of the theorem.

We now proceed with the proof.

I. The central ideas of the proof. Let $w_{0}, W_{1}, \cdots$ be a standard effective enumeration of the r.e. sets and let $\Phi_{0}, \Phi_{1}, \cdots$ be a standard effective enumeration of the partial recursive functionals. Define $c(i, X, n, s)$ to be the least number $c$ greater than $n$ such that if " $m \in X$ ?" is a question asked in the computation of $\Phi_{i}^{s}(X ; n)$ then $m \leq c$. Let $b$ be a recursive function from $N$ onto $N^{3}$. We define the triple $\left(\theta_{i}, \Psi_{i}, Y_{i}\right)$ to be the triple $\left(\Phi_{i_{0}}, \Phi_{i_{1}}, W_{i_{2}}\right)$ where $b(i)=$

$\left(i_{0}, i_{1}, i_{2}\right)$. We will construct a r.e. set $D$ in stages. The degree of $D$ will be the $\mathrm{d}$ satisfying the theorem. If $\bar{D} \neq W_{i}$ then we say that the nonrecursive condition of order $i$ is satisfied. If $\theta_{i}(D)=Y_{i}$ and $\Psi_{i}\left(Y_{i}\right)=D$ implies that there exist r.e. sets $Y_{i, 0}$ and $Y_{i, 1}$ such that $Y_{i, 0} \cap Y_{i, 1}=\varnothing, Y_{i, 0} \cup Y_{i, 1}=Y_{i}, D \leq_{T} Y_{i, 0}$, and $D \leq_{T} Y_{i, 1}$ then we say that the mitotic condition of order $i$ is satisfied.

Satisfying the nonrecursive condition of order $i$ has priority $2 i$, while satisfying the mitotic condition of order $i$ has priority $2 i+1$.

We define the following auxiliary functions. Let $b(i)=\left(i_{0}, i_{1}, i_{2}\right)$.

1. $L(i, s)=\mu m\left[\theta_{i}^{s}\left(D^{s} ; m\right) \neq Y_{i}^{s}(m)\right.$ or $\left.\Psi_{i}^{s}\left(Y_{i}^{s} ; m\right) \neq D^{s}(m)\right]$,

2. $j(i, n, s)=\max \left\{c\left(i_{0}, D^{s^{\prime}}, m, s^{\prime}\right): m \leq n, s^{\prime} \leq s\right\}$,

3. $k(i, n, s)=\max \left\{c\left(i_{1}, Y_{i}^{s^{\prime}}, m, s^{\prime}\right): m \leq n, s^{\prime} \leq s\right\}$,

4. $l(i, n, s)=$ the greatest $l<L(i, s)$ such that $j(i, l, s)<n$ if there is such a number $l$. If there is no such $l$ set $l(i, n, s)=-1$.

Remarks. (i) $l(i, n, s)<n \leq k(i, n, s)$. (ii) If $m \leq n$ then $j(i, m, s) \leq j(i, n, s)$, $k(i, m, s)<k(i, n, s)$, and $l(i, m, s) \leq l(i, n, s)$.

The proof is based on the following lemma.

Lemma. Let $i, x, y, s, s^{\prime}, s^{\prime \prime}$ be given sucb that $x \leq y, s \leq s^{\prime}<s^{\prime \prime}$. If

(i) $L(i, s)>k(i, y, s)$,

(ii) $L\left(i, s^{\prime}\right)>k(i, y, s)$ and $D^{s^{\prime}}(m)=D^{s}(m)$ for all $m \leq j(i, k(i, y, s), s)$,

(iii) $x \in D^{s^{\prime \prime}}-D^{s^{\prime}}, L\left(i, s^{\prime \prime}\right)>x, D^{s^{\prime \prime}}(m)=D^{s}(m)$ for all $m<x$,

then there exists $z \in Y_{i}^{s^{\prime \prime}}-Y_{i}^{s^{\prime}}$ sucb that $l(i, x, s)<z \leq k(i, y, s)$.

Proof. Since $D^{s^{n}}(m)=D^{s}(m)$ for all $m<x$ then $\theta_{i}^{s^{\prime \prime}}\left(D^{s^{n}} ; m\right)=\theta_{i}^{s}\left(D^{s} ; m\right)$ for for $m \leq l(i, x, s)$. If there exists $z \in Y_{i}^{s^{\prime \prime}}-Y_{i}^{s^{\prime}}$ such that $z \leq l(i, x, s)$ then cer- 
tainly $Y_{i}^{s^{\prime \prime}}(z) \neq \theta_{i}^{s^{\prime \prime}}\left(D^{s^{\prime \prime}} ; z\right)$. Hence $L\left(i, s^{\prime \prime}\right) \leq x$ contrary to the hypothesis. On the other hand suppose there exists no $z \leq k(i, y, s)$ such that $z \in Y_{i}^{s^{\prime \prime}}-Y_{i}^{s^{\prime}}$. Since $D^{s^{\prime}}(m)=D^{s}(m)$ for all $m \leq j(i, k(i, y, s), s)$ then $\theta_{i}^{s^{\prime}}\left(D^{s^{\prime}} ; m\right)=\theta_{i}^{s}\left(D^{s} ; m\right)$ for all $m \leq k(i, y, s)$. Since $L\left(i, s^{\prime}\right)>k(i, y, s)$ then we must have $Y_{i}^{s^{\prime}}(m)=$ $Y_{i}^{s}(m)$ for all $m \leq k(i, y, s)$. Hence we have that there is no $z \leq k(i, y, s)$ such that $z \in Y_{i}^{s^{\prime \prime}}-\overline{Y_{i}^{s}}$ which implies that $\Psi_{i}^{s^{\prime \prime}}\left(Y_{i}^{s^{\prime \prime}} ; m\right)=\Psi_{i}^{s}\left(Y_{i}^{s} ; m\right)$ for all $m \leq y$. Since $x \in D^{s^{\prime \prime}}-D^{s^{\prime}}$ then $D^{s^{\prime \prime}}(x) \neq \Psi_{i}^{s^{\prime \prime}}\left(Y_{i}^{s^{\prime \prime}} ; x\right)$. Hence $L\left(i, s^{\prime \prime}\right) \leq x$ contrary to the hypothesis.

Figure 1 illustrates the lemma.

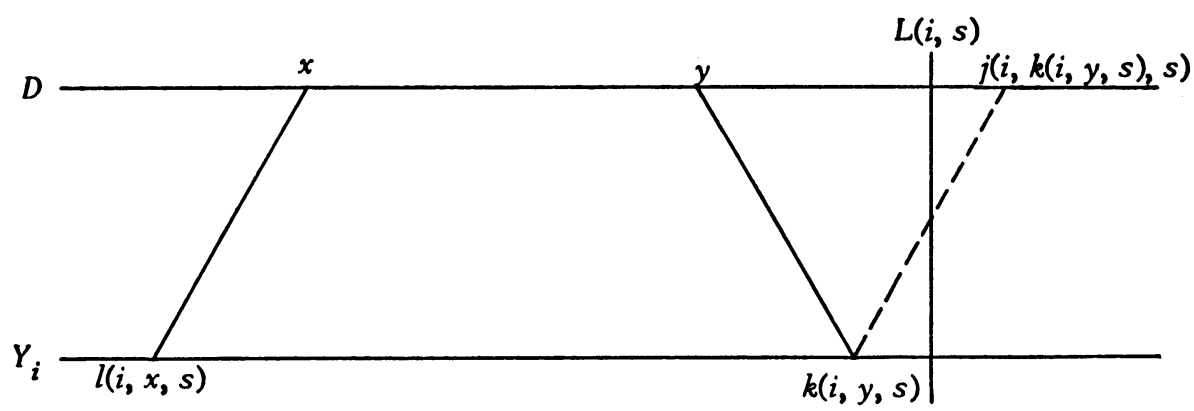

Figure 1

Notice that $\lim _{s} L(i, s)=\infty$ iff $\theta_{i}(D)=Y_{i}$ and $\Psi_{i}\left(Y_{i}\right)=D$. The basic lemma tells us how to indirectly control $Y_{i}$ by controlling $D$. If $\theta_{i}(D)=Y_{i}$ and $\Psi_{i}\left(Y_{i}\right)=D$ then by the lemma we can force a number into $Y_{i}$ in a specific interval if we put a number into $D$.

One mitotic condition. It is helpful to explain how to satisfy one mitotic condition, say the first mitotic condition, while satisfying all the nonrecursive conditions. For this purpose we shall only need a special case of the lemma, the case when $x=y$. In order to make $D$ nonrecursive we may want to put relatively small numbers into $D$. Each time we do so we would like to force two numbers into $Y_{0}$ one for each of the two sets $Y_{0,0}$ and $Y_{0,1}$. Of course, we need only do this if it looks like $L(0, s)$ is becoming large, that is, if it looks like it may be the case that $\theta_{0}(D)=Y_{0}$ and $\Psi_{0}\left(Y_{0}\right)=D$. Assume $L(0, s)$ is large and we want to put $n$ into $D$ to satisfy some nonrecursive condition. By the lemma we can put $n$ into $D$ and force some number into $Y_{0}$ in a specific interval. Waiting in the wings is another number $m$ which we will put into $D$ to force another number into an interval disjoint from the first. Figure 2 helps illustrate this procedure. 


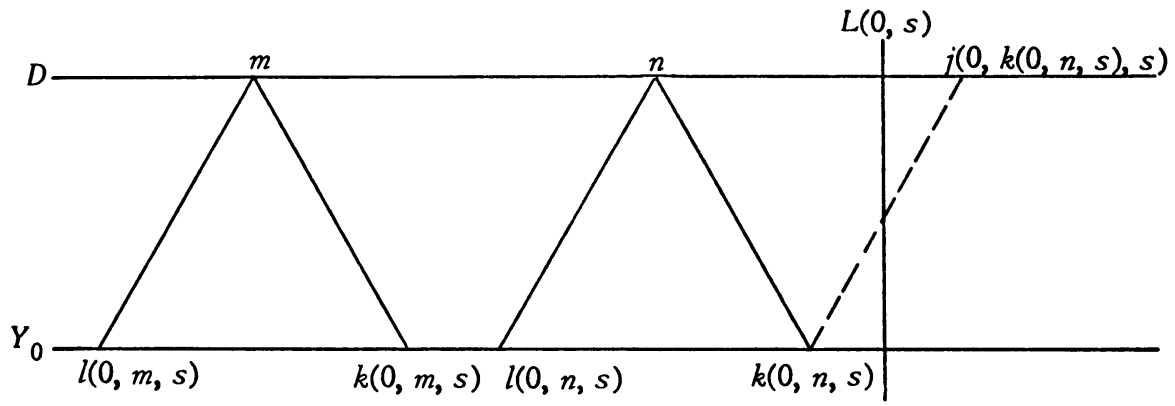

Figure 2

Choose $m$ and $n$ such that $L(0, s)>k(0, n, s)$ and $k(0, m, s) \leq l(0, n, s)$. Suppose $n$ is ready to satisfy a nonrecursive condition at stage $s_{0}+1>s$. We assume that no number $\leq j(0, k(0, m, s), s)$ was put into $D$ after stage $s$ and before stage $s_{0}+1$. We put $n$ into $D$ at stage $s_{0}+1$ and wait for a stage $s_{1}>$ $s_{0}+1$ such that $L\left(0, s_{1}\right)>n$. At stage $s_{1}+1$ we put $m$ into $D$. Let $s_{2}$ be the least stage $>s_{1}+1$ such that $L\left(0, s_{2}\right)>m$. By the lemma we can conclude that there are two numbers $y_{0}$ and $y_{1}$ such that $y_{0}, y_{1} \in Y_{0}^{s}-Y_{0}^{s}, l(0, m, s)<y_{0} \leq$ $k(0, m, s)$ and $l(0, n, s)<y_{1} \leq k(0, n, s)$. We may now put $y_{0}$ into $Y_{0,0}$ and $y_{1}$ into $Y_{0,1}$. This procedure depends on the existence of stages $s_{1}$ and $s_{2}$. However if one of $s_{1}$ and $s_{2}$ does not exist then the mitotic condition is satisfied vacuously since $\lim _{s} L(i, s) \neq \infty$. The preceding explains how to get two different numbers into $Y_{0}$ but only locally.

We now give a global picture of how to construct $D$ just satisfying one mitotic condition. Candidates are chosen to follow nonrecursive conditions. The candidate of order $i$ follows $\bar{D} \nRightarrow W_{i}$. Candidates are of two types, members of $N$ (type 0 ) and members of $N \times N$ (type 1). Actually only the second coordinate of a member of $N \times N$ is used to satisfy a nonrecursive condition but it is easier to think of the pair as the candidate. In general an unsatisfied nonrecursive condition starts off with a type 0 candidate and then tries to attain a type 1 candidate. As $D$ is constructed so are sets $E, A, R_{0}$, and $R_{1}$, and functions $d$ and r. We shall always have $A^{s} \subseteq E^{s} \subseteq N \times N, R_{0}^{s} \cap R_{1}^{s}=\varnothing$ and $r(s)=$ $\max \left(R_{1}^{s} \cup\{-1\}\right)$. Figure 3 illustrates a stage $s$ in the construction.

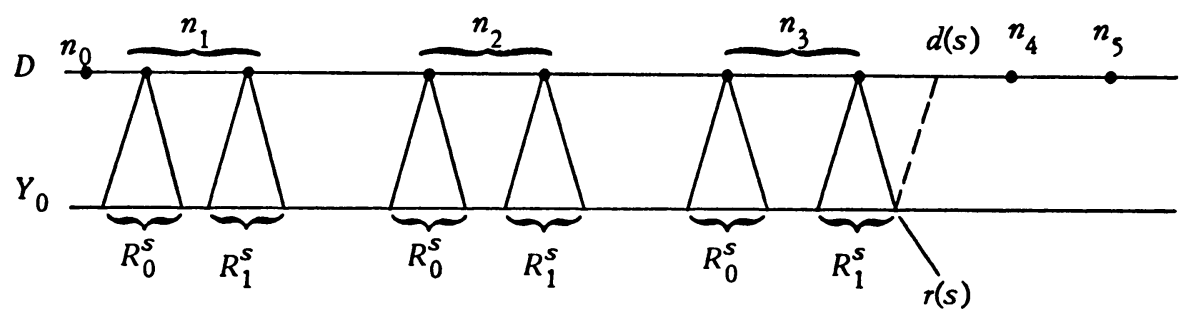

Figure 3 
The point $n_{i}$ is the candidate of order $i$ at stage $s$ and $A^{s}=E^{s}=\left\{n_{1}, n_{2}, n_{3}\right\}$. Notice that the first nonrecursive condition has only a type 0 candidate and will never attain a type 1 candidate. This is because the first nonrecursive condition is of higher priority than the first mitotic condition. If and when $n_{0}$ is put into $D$ then the entire picture is "erased", that is, we set $A^{s}=E^{s}=\varnothing, R_{0}^{s}=R_{1}^{s}=$ $\varnothing, d(s)=-1$, and cancel all candidates. Several things can happen at stage $s+1$.

1. A candidate is chosen. No candidate for $\bar{D} \nRightarrow W_{6}$ exists, so one of type 0 is chosen at stage $s+1$ which is larger than $d(s)$ and larger than any candidate of higher priority.

2. A candidate is improved. The condition $\bar{D} \neq W_{4}$ has a candidate of type 0 and can gain a candidate of type 1 . There exists $p, q \in \bar{D}^{s}$ such that $r(s)<$ $l(0, p, s), k(0, p, s)<l(0, q, s)$, and $L(0, s)>k(0, q, s)$. The new candidate of order 4 becomes $n_{4}=(p, q)$ and candidates of higher order are cancelled. We then have $A^{s+1}=E^{s+1}=\left\{n_{1}, n_{2}, n_{3}, n_{4}\right\}, R_{0}^{s+1}=R_{0}^{s} \cup[l(0, p, s), k(0, p, s)]$, $R_{1}^{s+1}=R_{1}^{s} \cup[l(0, q, s), k(0, q, s)]$ and $d(s+1)=j(0, k(0, q, s), s)$. Figure 4 illustrates the situtation at stage $s+1$.

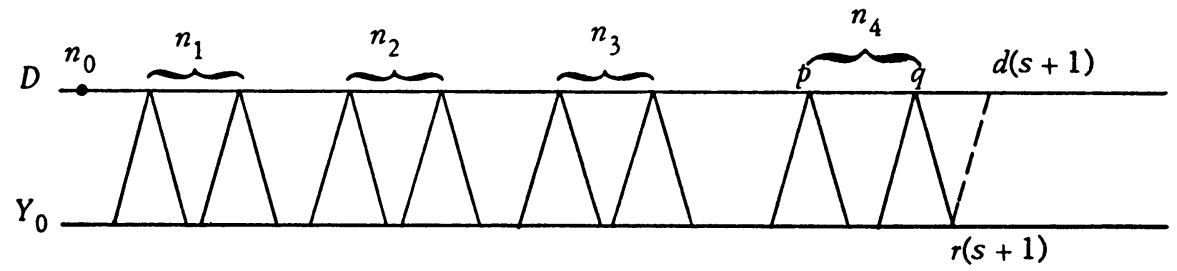

Figure 4

3. A candidate is implemented. We look at three cases. First suppose $n_{0} \epsilon$ $w_{0}^{s+1}$. As was mentioned earlier we put $n_{0}$ into $D$ at stage $s+1$ and "erase" everything. The second case is when a type 0 candidate other than $n_{0}$ is implemented, for instance suppose $n_{4} \in W_{4}^{s+1}$. Just put $n_{4}$ into $D$ and relax for we have satisfied a nonrecursive condition without any need to worry about mitoticity. The third case is when we implement a type 1 candidate. Let $n_{2}=(p, q)$ and suppose $q \in W_{2}^{s+1}$. Put $q$ into $D$. We now set up a potential condition of order 2 which consists of the point $(p, q)$. The potential condition will be satisfied at stage $s^{\prime}$ if $L\left(0, s^{\prime}\right)>q$. We have $E^{s+1}=E^{s}$ but $A^{s+1}=\left\{n_{1}\right\}$. Further $d(s+1)=d(s), R_{0}^{s+1}=R_{0}^{s}$, and $R_{1}^{s+1}=R_{1}^{s}$. Notice that the point $n_{3}$ is no longer any good because a number less than it has entered $D$. The set $A^{s}$ is the set of points that are still good. Cancel all candidates and potential conditions of order $>2$. Figure 5 illustrates the situation at stage $s+1$. 


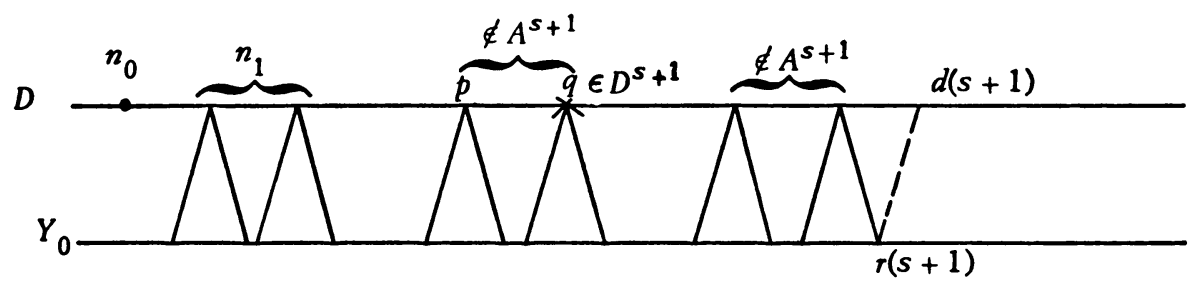

Figure 5

4. A potential condition is satisfied. Let us consider a stage $s^{\prime} \geq s+1$ (stage $s+1$ as pictured in Figure 5). At stage $s+1$ the potential condition $(p, q)$ was instituted. Suppose $L\left(0, s^{\prime}\right)>q$, that is, the potential condition $(p, q)$ is satisfied at stage $s^{\prime}$. At stage $s^{\prime}+1$ put $p$ into $D$. (There is no need now to set up another potential condition because we do not need to put a smaller than $p$ into $D$.) Cancel all candidates of order $>2$.

The four situations we have just described are organized as follows. At stage $s+1$ find the least $i$ such that one of the following holds:

A. The potential condition of order $i$ is satisfied at stage $s$.

B. The candidate of order $i$ can be implemented at stage $s$ and $D^{s} \cap w_{i}^{s}=\varnothing$.

C. The candidate of order $i$ can be improved at stage $s$.

D. A candidate of order $i$ can be chosen at stage $s$ and $D^{s} \cap W_{i}^{s}=\varnothing$.

Now at stage $s+1$ do the appropriate task described above in 1-4.

It should be clear that all the nonrecursive conditions are satisfied. Now suppose $\theta(D)=Y_{0}$ and $\Psi\left(Y_{0}\right)=D$. Since $\lim _{s} L(0, s)=\infty$ then we must have constructed infinitely many type 1 points. Hence we must have $\lim _{s} d(s)=\infty$. Notice there is at most one stage $t$ such that $E^{t} \underline{\not} E^{t+1}$ ( $n_{0}$ enters $D$ at stage $t+1$ ). Let $t_{0}$ be the least stage such that $E^{s} \subseteq E^{s+1}$ for all $s \geq t_{0}$. Define

$$
\begin{aligned}
R & =\bigcup_{s \geq t_{0}} R_{0,}^{s}, \quad Y_{0,0}^{s}=Y_{0}^{s} \cap R, \quad Y_{0,1}^{s}=Y_{0}^{s} \cap \bar{R} \\
Y_{0,0} & =\bigcup_{s} Y_{0,0}^{s} \quad \text { and } Y_{0,1}=\bigcup_{s} Y_{0,1}^{s}
\end{aligned}
$$

Now, $R$ is recursive since its intervals are enumerated in increasing order. Hence $Y_{0,0}$ and $Y_{0,1}$ are r.e. Clearly $Y_{0}=Y_{0,0} \cup Y_{0,1}$ and $Y_{0,0} \cap Y_{0,1}=\varnothing$. We show now how to reduce $D$ to $Y_{0,0^{\circ}}$ A similar argument will show $D \leq_{T} Y_{0,1^{\bullet}}$

Let $x$ be a fixed argument. We can assume inductively that we know $D(y)$ for all $y<x$. Let $t_{1}+1$ be the least stage $\geq t_{0}$ such that $d\left(t_{1}+1\right) \geq x$. Let $t_{2}$ be the least stage $\geq t_{1}+1$ such that 
(i) $D(y)=D^{t} 2(y)$ for all $y<x$,

(ii) $Y_{0,0}(y)=Y_{0,0}^{t_{2}}(y)$ for all $y \leq r\left(t_{1}+1\right)$,

(iii) $L\left(0, t_{2}\right)>r\left(t_{1}+1\right)$,

(iv) no potential condition exists at stage $t_{2}$.

Now, $x \in D$ iff $x \in D^{t}$.

To argue this suppose $x \in D-D^{l} 2$. First of all $x$ must be the first coordinate of a type 1 point created at stage $t_{1}+1$. If $x$ were the second coordinate then at some time after $x$ was put into $D$ a number smaller than $x$ would be put into $D$, otherwise, a potential condition would be in existence forever. At stage $t_{1}+1$ we have the situation described in Figure 6.

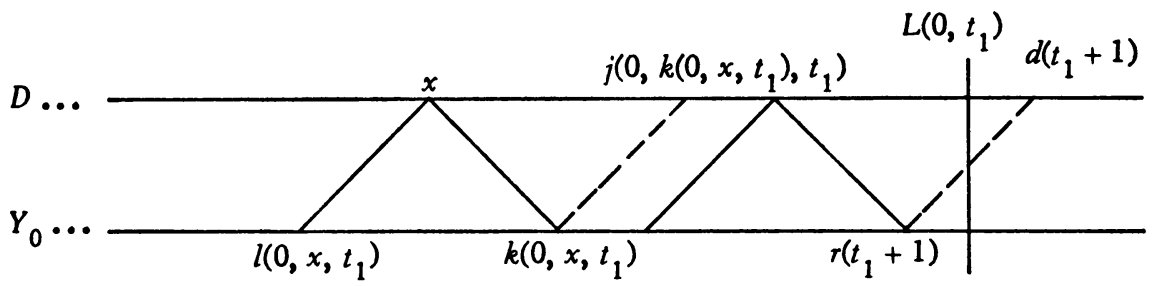

Figure 6

We certainly have $L\left(0, t_{1}\right)>k\left(0, x, t_{1}\right)$. At stage $t_{2}$ we must have $L\left(0, t_{2}\right)>$ $k\left(0, x, t_{1}\right)$ and $D^{t^{1}}(y)=D^{t_{2}}(y)$ for all $y \leq j\left(0, k\left(0, x, t_{1}\right), t_{1}\right)$. There must be a stage $t_{3}>t_{2}$ such that $x \in D^{t_{3}}$ and $L\left(0, t_{3}\right)>x$. By the basic lemma there exists a $z \in Y_{0}^{t}{ }^{3}-Y_{0}^{t}{ }^{2}$ such that $l\left(0, x, t_{1}\right)<z \leq k\left(0, x, t_{1}\right)$. Hence $z \in Y_{0,0}-Y_{0,0}^{t^{2}}$ and $z \leq r\left(t_{1}+1\right)$ which is impossible.

There is an important fact about the construction which should be noted. The mitotic condition is never satisfied once and for all at any finite stage. We must continually work on satisfying the mitotic condition. This kind of thing happens in the standard maximal set construction. If one considers a maximal condition as satisfied if $\bar{M} \cap W_{i}$ or $\bar{M} \cap \bar{W}_{i}$ is finite then these conditions are not satisfied at any finite stage but must continually be worked on. A useful tool in such constructions is the concept of "e-state" introduced by Yates [5]. Although we shall not mention "e-state" explicitly in our construction the idea is implicit in our notion of "type". The difficulties that arise in the construction may be best illustrated by indicating how to construct $D$ satisfying just two of the mitotic conditions, say the first two.

Two mitotic conditions. In satisfying two mitotic conditions there are four types of points to be considered. Figure 7 illustrates these types of points. 


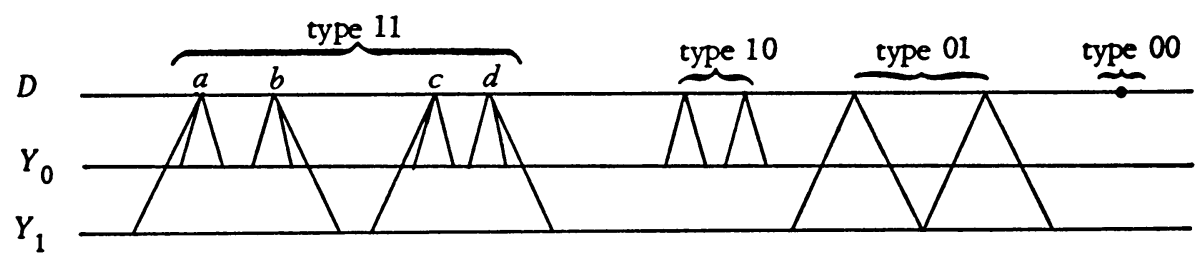

Figure 7

Notice that type 11 points are built up from type 10 points. The basic strategy in constructing $D$ can be summarized as follows:

Satisfy $\bar{D} \neq W_{i}$ with an $\alpha$ point $(\alpha=11,10,01,00)$ where $\alpha$ is greatest lexicographically.

We have already seen how 10,01 , and 00 points work. Type 11 points are constructed to enable us to satisfy the two mitotic conditions simultaneously. Let us examine the point $((a, b),(c, d))$ of Figure 7 . Suppose $d$ is ready to satisfy a nonrecursive condition at stage $s_{0}$. Put $d$ into $D^{s_{0}+1}$ and wait for a stage $s_{1}$ when $L\left(0, s_{1}\right)>d$. Now put $c$ into $D^{s_{1}+1}$ and wait for a stage $s_{2}$ when $L\left(0, s_{2}\right)>c$. At this point housekeeping on the first mitotic condition is in order. Now wait further until $L\left(1, s_{3}\right)>c$. Now put $b$ into $D^{s_{3}+1}$ and wait until a stage $s_{4}$ when $L\left(0, s_{4}\right)>b$. Now put $a$ into $D^{s_{4}+1}$ and we are finished. A more sophisticated potential condition is used to keep track of how the point is progressing. It is important to notice that the second mitotic condition does not hold up the satisfaction of the first. While we are waiting for stage $s_{3}$ which concerns the second mitotic condition we are not in the middle of a point trying to satisfy the first mitotic condition. On the other hand we do allow the first mitotic condition to hold up satisfaction of the second. If the first mitotic condition holds up satisfaction of the second it does so only on a 11 point. We may now fall back on 01 points in order to satisfy the second mitotic condition.

Rather than describe a construction of $D$ satisfying two mitotic conditions we shall just mention the difficulties that arise in satisfying two conditions and how we overcome them. It may be helpful to refer back to these explanations when reading the construction and proof.

(1) The order of candidates. In general our strategy is to try to satisfy a nonrecursive condition with the highest possible type point. To implement this we must always make sure that if $i<j, p$ is the candidate of order $i$ and $q$ is the candidate of order $j$ then $p$ lies to the left of $q$. So if $q$ is implemented then $p$ is still good and can be implemented later.

(2) The order of types of points. Also to implement our strategy we must make sure that the lowest order candidates have the highest type points. How- 
ever, 11 points are built up from 10 points hence we cannot say that 11 points must lie to the left of 10 points. These two types are compatible. On the other hand 10 points and 01 points are incompatible and 01 points must always lie to the right of 10 points. Let Figure 8 illustrate a situation at stage $s$.

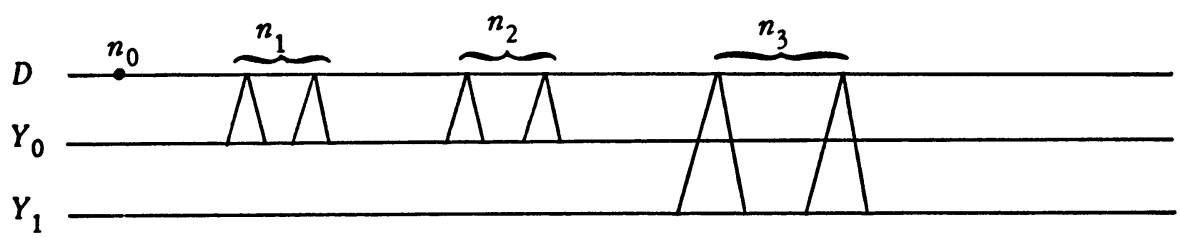

Figure 8

At stage $s+1$ we may improve the candidate of order 3 to be a type 10 point. What do we do with the present candidate? Completely erase the point to get the situation illustrated in Figure 9.

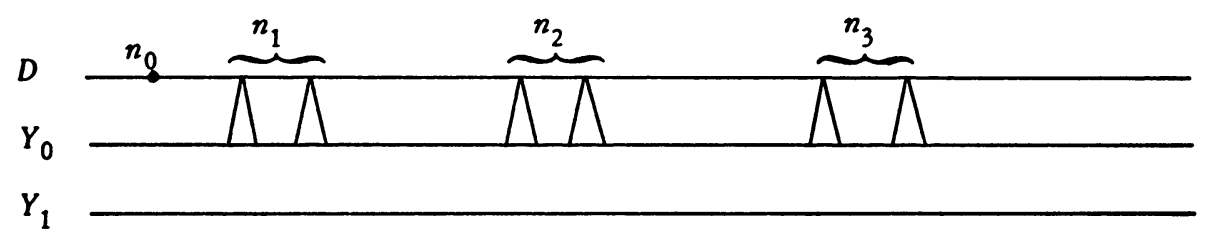

Figure 9

Although we may have forsaken the second mitotic condition it is only temporarily. If this situation keeps repeating we shall eventually be able to create 11 points which are better than 01 points.

(3) An unbounded store of good points. In order to create 11 points from 10 points we must be sure that we maintain an ever increasing store of good 10 points to build from. To do this we use the fact that there are infinitely many $i$ such that $w_{i}=\varnothing$ (candidates of these orders are never implemented). We never use the first $i$ good 10 points in constructing a type 11 candidate of order $i$.

(4) A conflict between implementation and improvement. Consider the situation at stage $s$ illustrated in Figure 10.

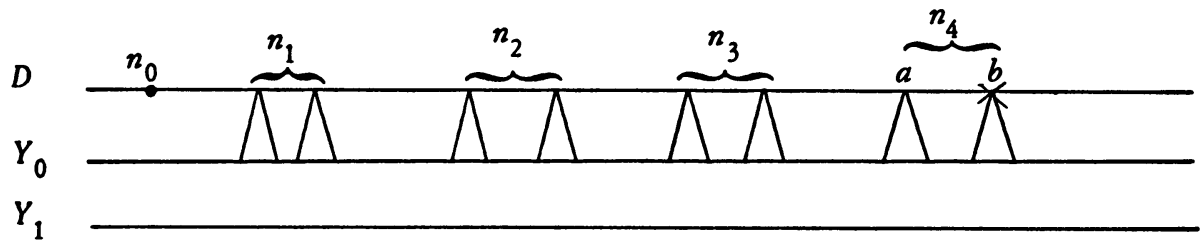

Figure 10 
The number $b$ is presently in $D$ and we are waiting for $L(0, s)$ to exceed $b$ in order to put $a$ into $D$. However at stage $s+1$ we could construct a type 11 candidate using $n_{2}$ and $n_{3}$. Properly speaking this new candidate should be the candidate of order 2 replacing $n_{2}$. This leaves the condition $\bar{D} \vDash W_{3}$ without a candidate. However the condition $\bar{D} \neq W_{4}$ would still have a candidate. The order of candidates is ruined. To resolve this problem we do not form the type 11 candidate at this time. While we are waiting for $L(0, s)$ to exceed $b$ we can think of the mitotic condition as temporarily satisfied for if $L(0, s)$ never exceeds $b$ then it is satisfied. Hence there is no need at the moment for type 11 points to be constructed. While we are waiting for $L(0, s)$ to exceed $b$ we shall say 10 and 11 are frozen.

(5) A conflict between frozen types and improvement. This conflict is best illustrated using three mitotic conditions. Suppose we have the following situation at stage $s$ illustrated in Figure 11.

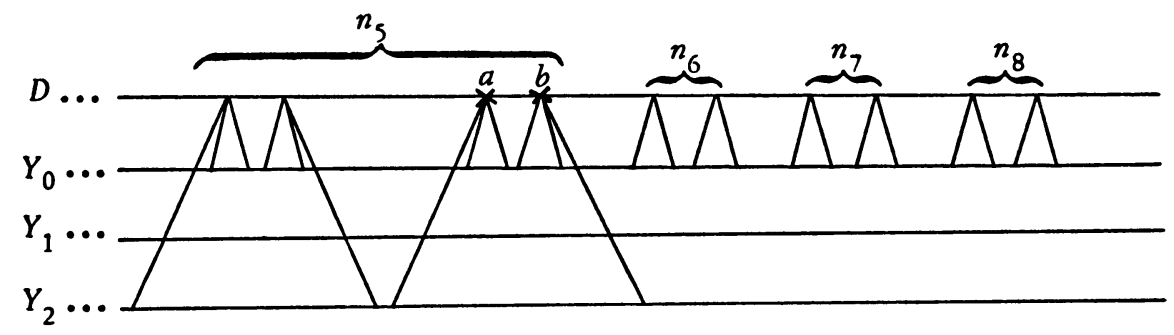

Figure 11

At stage $s$ both $a$ and $b$ have been put into $D$ and we are waiting. Now type 101 is frozen but not type 100. Suppose at stage $s+1$ we could improve the candidate of order 6 to be a 110 point using $n_{6}$ and $n_{8}$ as subpoints. We go ahead and do just that to get the situation illustrated in Figure 12.

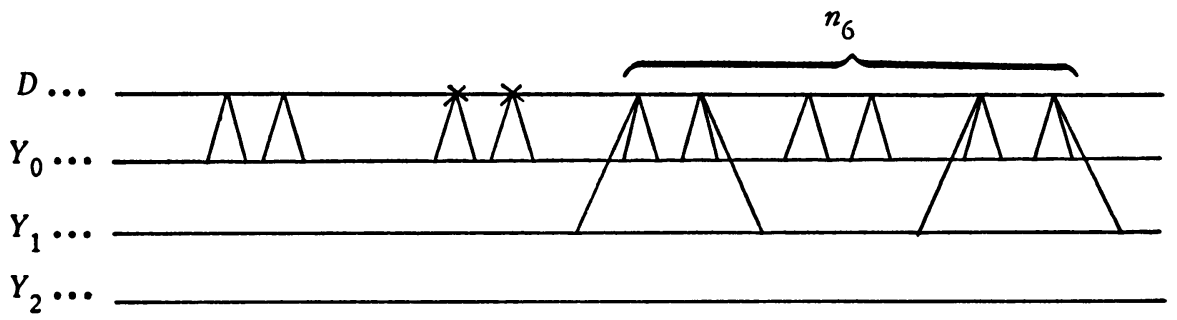

Figure 12

The candidate of order 5 goes out of existence. You may ask how can improving the candidate of order 6 have priority over continuing to implement the candidate 
of order 5 ? When we put $b$ into $D$ we satisfied the condition $\bar{D} \neq W_{S}$ once and for all. The rest of the implementation of the candidate of order 5 is concerned with satisfying mitotic conditions. Thus we have not really violated the priorities at all but have adhered to them in constructing a 110 point over a 101 point.

The list (1)-(5) is not an exhaustive list of all the difficulties in the construction but only contains what we deem to be the most important. We now proceed with the formal proof.

II. Notation and definitions. Define $N^{[0]}=N$ and $N^{[k+1]}=N^{[k]} \times N^{[k]}$. We let $P=\bigcup\left\{N^{[k]}: k \in N\right\}$ and call the members of $P$ points. If $p \in N^{[k]}$ then we write $l h p=k$. Let $\Sigma$ be the set of all finite sequences of 0 's and 1's. We denote the empty sequence by $\lambda$, the length of a sequence $\sigma$ by $l b \sigma$, and the concatenation of two sequences $\sigma$ and $r$ by $\sigma * \tau$. For $n<l b \sigma$ the $(n+1)$ st member of the sequence $\sigma$ is denoted by $\sigma_{n}$. If $l b \sigma=l b r$ then $\sigma<\tau$ just in case $\sigma \neq \tau$ and if $n=\mu m\left(\sigma_{m} \neq r_{m}\right)$ then $\sigma_{n}=0$. If $p \in P$ then $(p)_{0}$ and $(p)_{1}$ are the first and second coordinates of $p$ respectively. If $l h \sigma \leq l h p$ then we define $(p)_{\sigma}$ inductively on the length of $\sigma$. We let $(p)_{\lambda}=p$ and $(p)_{\sigma * i}=\left((p)_{\sigma}\right)_{i}$ where $i$ is 0 or 1 . For example, if $p=((1,2)(3,4))$ then $(p)_{0}=(1,2),(p)_{1}=(3,4),(p)_{00}=1,(p)_{01}=2$, $(p)_{10}=3$, and $(p)_{11}=4$. We define $(p)_{0}=(p)_{\sigma}$ where $\sigma$ is the constant zero sequence of length equal to $l b p$. Likewise $(p)_{i}$ is defined. If $p, q \in P$ then we write $p<q$ just in case $(p)_{\overline{1}}<(q)_{\overline{0}}$.

Let $\Gamma$ be the set of all eventually zero infinite sequences of 0 's and 1 's. Members of $\Gamma$ are used to classify points into types. When we were examining just two mitotic conditions a type was a sequence of length two but now that we are dealing with infinitely many mitotic conditions our types are infinite. If $a \in \Gamma$ then $a_{n}$ denotes the $(n+1)$ st member of the sequence $a$. We denote the constant zero sequence by the symbol $o$, omicron. We consider two partial orders on $\Gamma$.

1. The lexicograpbic order: $\alpha \leq \beta$ if $\alpha=\beta$ or $\left[\alpha \neq \beta\right.$ and if $n=\mu n^{\prime}\left(\alpha_{n}^{\prime \prime} \neq \beta_{n}{ }^{\prime}\right)$ then $a_{n}=0$ ].

2. The tree order: $\alpha \subseteq \beta$ if there exists $n$ such that, for all $m<n, a_{m}=\beta_{m}$ and, for all $m \geq n, a_{m}=0$.

We define (level of $\alpha) \operatorname{lev} \alpha=\mu n\left(\forall m \geq n, a_{m}=0\right)$. Thus levo=0. Further, we define $\alpha * n$ to be the sequence $\beta$ where $\beta_{m}=\alpha_{m}$ if $m \neq n$ and $\beta_{n}=1$. Notice that if $n \geq \operatorname{lev} \alpha$ then lev $\alpha * n=n+1$ and $\alpha \subseteq \beta$ if and only if $\alpha_{n}=\beta_{n}$ for all $n<\operatorname{lev} \alpha$. We define (extent of $\alpha$ ) ext $\alpha=\operatorname{Card}\left\{n: \alpha_{n}=1\right\}$.

For each $a \in \Gamma$ we will construct sets $E_{a}, A_{a}, R_{0, a}$ and $R_{1, a}$. These sets will be constructed in stages. We will always have $A_{a}^{s} \subseteq E_{a}^{s} \subseteq P$. The order on $P$ always induces an order on $E_{a}^{s}$ isomorphic to an initial segment of the natural numbers. 


$$
\begin{aligned}
n(j, a, s) & =\left\{\begin{array}{l}
\text { the }(j+1)_{s t} \text { member of } A_{a}^{s} \text { if Card } A_{a}^{s} \geq j+1, \\
\text { the last member of } A_{a}^{s} \text { if } 0<\operatorname{Card} A_{a}^{s} \leq j, \\
0 \text { if } A_{a}^{s}=\varnothing,
\end{array}\right. \\
m(j, a, s) & =\max \left\{\left(n\left(j, \beta, s^{\prime}\right)\right)_{i}: \beta \leq a \text { and } s^{\prime} \leq s\right\}, \\
r(a, s) & =\max \left(R_{1, a}^{s} \cup\{-1\}\right) .
\end{aligned}
$$

We shall also construct a function $\lambda a s d(a, s)$ with the property that if $a \neq o$ then $d(\alpha, s)>\max E_{\alpha}^{s}$. We define

$$
d(s)=\max \{d(\alpha, s): \alpha \in \Gamma\} .
$$

At various stages $s$ we may appoint a point $p$ to be the candidate of order $i$ for some $i$. The point $p$ will initially be a member of $A_{a}^{s}$ for some $a$ where lev $a \leq i$. If $p$ is appointed at stage $s$ to be the candidate of order $i$ and $p \in A_{a}^{s}$ then we say $p$ has type $a$. If the candidate of order $i$ in existence at stage $s$ is such that $(p)_{-} \in D^{s}$ then the candidate is said to be implemented and a potential condition of order $i$ must also be in existence. A potential condition associated with a candidate $p$ of type $a$ is of the form $(p, \alpha, \sigma, j)$ where $\sigma \in \Sigma$ and $j \in N$. We say that the potential condition, $(p, \alpha, \sigma, j)$, is satisfied at stage $s$ if $L(j, s)>$ $(p)_{\sigma}$. For each $i$ and $s$ there is at most one candidate of order $i$ and at most one potential condition of order $i$ in existence at stage $s$. Once a candidate or potential condition is appointed it remains in existence until cancelled or changed. If an implemented candidate is cancelled at stage $s$ then so also is the potential condition associated with it. It is important to notice that if a candidate $p$ of type $a$ is in existence at stage $s$ that does not by definition imply that $p \in E_{a}^{s}$. The type of a candidate refers to its initial status when appointed. However, we shall show in Claim 1 that if a candidate in existence at stage $s$ has type $a$ then it is indeed a member of $E_{a}^{s}$.

We say that $\alpha$ is frozen at stage $s$ if there exists a potential condition of the form $(p, \beta, \sigma, i)$ such that if $\gamma$ is the greatest member of $\Gamma$ such that $\gamma \subseteq \beta$ and $\gamma \subseteq \alpha$ and $\sigma=\sigma^{\prime} * \sigma^{\prime \prime}$ where $l b \sigma^{\prime \prime}=\operatorname{ext} \gamma$ then $\sigma^{\prime \prime}$ has a nonzero member. The notion of being frozen is explained in our list of difficulties (4) and (5). We should note that if $\alpha$ is frozen at stage $s$ and $\alpha \subseteq \delta$ then $\delta$ is also frozen at stage $s$. Further if a potential condition of the form $(p, \beta, \sigma, i)$ exists at stage $s$ and if $\beta \subseteq \alpha$ then $\alpha$ is frozen at stage $s$. Basically $\alpha$ is frozen at stage $s$ if we are presently implementing a candidate such that unless the potential condition associated with that candidate is satisfied at some time then only finitely many points of type a could ever be constructed because $\lim _{s} L(j, s) \neq \infty$ for some $j$ such that $\alpha_{j}=1$. 
The candidate of order $i$ can be improved at stage $s+1$ if all the following hold:

(1) an unimplemented candidate $r$ of order $i$ already exists at stage $s$,

(2) $r$ has type $\alpha$ and there exists $j, \beta, p, q$ such that

(a) $p \in A_{\beta}^{s}$ and $q \in A_{\beta}^{s}$,

(b) $p<q$,

(c) $\operatorname{lev} \beta \leq j<i$,

(d) $\beta \subseteq \alpha$ and $\beta * j>\alpha$,

(e) $m(i, \beta, s)<p$,

(f) $\beta * j$ is not frozen at stage $s$,

(g) $p>$ any unimplemented candidate of order $<i$ in existence at stage $s$,

(h) $L(j, s)>k\left(j,(q)_{-}, s\right)$,

(i) $r(\gamma, s)<l\left(j,(p)_{0}, s\right)$ for all $\gamma \geq \beta * j$,

(j) $k\left(j,(p)_{-}, s\right)<l\left(j,(q)_{-}, s\right)^{\text {. }}$

If indeed we do improve the candidate of order $i$ to be the point $(p, q)$ at stage $s+1$ then we may explain the reasons for $(a)-(j)$ by $\left(a^{\prime}\right)-\left(j^{\prime}\right)$ below.

$\left(a^{\prime}\right)(p, q)$ will be a "good" point of type $\beta * j$.

$\left(b^{\prime}\right)$ If implemented the coordinates of $(p, q)$ will enter $D$ in descending order.

(c') This insures that the candidate of order $i$ can have only one of $2^{i}$ types.

(d') $\beta \subseteq a$ insures that there must have been a candidate of order $i$ and type $\beta$ before one of type $\beta * j$ was chosen while $\beta * j>a$ insures that $(p, q)$ is of higher type than $r$.

(e') This will insure that, for each $\delta \subseteq \beta, A_{\delta}^{s}$ will become unbounded if $E_{\delta}^{s}$ becomes unbounded (see difficulty (3)).

$\left(f^{\prime}\right)$ (See difficulties (4) and (5) and the definition of frozen.)

$\left(\mathrm{g}^{\prime}\right)$ This is to insure the ordering of the candidates is correct. We only require that $p>$ any unimplemented candidates of order $\langle i$ not $>$ all candidates of order $<i$ for the following reason. An implemented candidate may be of type $<\beta * j$ and will be cancelled as soon as $(p, q)$ is created. After all, the nonrecursive condition associated with this low type candidate is already satisfied so there is no need to keep low type candidates around that will hold up the creation of high type candidates (difficulty (5) goes into this problem somewhat).

$\left(h^{\prime}\right)-\left(j^{\prime}\right)$ We have gone into this extensively when we considered one mitotic condition.

The condition of order $i$ requires attention at stage $s+1$ if one of the following holds:

A. The potential condition of order $i$ is satisfied at stage $s$.

B. $D^{s} \cap W_{i}^{s}=\varnothing$ and the candidate $p$ of order $i$ is such that $(p)_{1} \in W_{i}^{s+1}$.

C. The candidate of order $i$ can be improved at stage $s+1$.

D. $D^{s} \cap W_{i}^{s}=\varnothing$ and there is no candidate of order $i$ in existence at stage $s$. 
III. The construction.

Stage 0 .

$$
\begin{gathered}
D^{0}=\varnothing, \quad d(\gamma, 0)=-1, \quad A_{o}^{0}=E_{o}^{0}=N, \\
A_{\gamma}^{0}=E_{\gamma}^{0}=\varnothing \quad \text { if } \gamma \neq o, \quad R_{0, \gamma}^{0}=R_{1, \gamma}^{0}=\varnothing .
\end{gathered}
$$

Stage $s+1$. Choose the condition of least order that requires attention at stage $s+1$. If none go to the next stage. We adopt the case that corresponds to the first of the four possibilities that holds.

A. Let $(p, a, \sigma, j)$ be the potential condition of order $i$ satisfied at stage $s$. Let $n=1+$ the number of zeros at the end of $\sigma$.

Case 1. $j<$ the $n$th $k$ such that $\alpha_{k}=1$. In this case make no changes except that the potential condition of order $i$ becomes $(p, \alpha, \sigma, k)$ where $k$ is the least number $>j$ such that $\alpha_{k}=1$.

Case 2. $j=$ the $n$th $k$ such that $a_{k}=1$. Cancel all candidates and potential conditions of order $>i$. Let $\sigma^{\prime}$ be the immediate predecess or of $\sigma$ in the < ordering of $\boldsymbol{\Sigma}$.

$$
\begin{gathered}
D^{s+1}=D^{s} \cup\left\{(p)_{\sigma^{\prime}}\right\}, \quad d(\beta, s+1)= \begin{cases}d(\beta, s) & \text { if } \beta \geq \alpha \text { or } \beta \subseteq \alpha, \\
-1 & \text { otherwise, }\end{cases} \\
E_{\beta}^{s+1}=\left\{\begin{array}{ll}
E_{\beta}^{s} \text { if } \beta \geq a \text { or } \beta \subseteq \alpha, \\
\varnothing \quad \text { otherwise, }
\end{array} R_{j, \beta}^{s+1}= \begin{cases}R_{j, \beta}^{s} & \text { if } \beta \geq \alpha \text { or } \beta \subseteq \alpha, \\
\varnothing \quad \text { otherwise, }\end{cases} \right. \\
A_{\beta}^{s+1}=\left\{\begin{array}{l}
\left\{r \in A_{\beta}^{s}: r<p\right\} \cup\left\{r \in \bar{D}^{s+1}: r>d(s+1)\right\} \text { if } \beta=o, \\
\left\{r \in A_{\beta}^{s}: r<p\right\} \text { if } \beta \neq o \text { and }(\beta \subseteq \alpha \text { or } \beta \geq \alpha), \\
\varnothing \text { otherwise. }
\end{array}\right.
\end{gathered}
$$

If $\sigma^{\prime}$ is not a constant zero sequence then the potential condition of order $i$ becomes $\left(p, a, \sigma^{\prime}, k\right)$ where $k$ is the least number such that $\alpha_{k}=1$. If $\sigma^{\prime}$ is a constant zero sequence then cancel the candidate of order $i$. Hence the potential condition of order $i$ is also cancelled.

Case 3. Neither Case 1 nor 2 applies. Go to the next stage. (We shall show in Claim 2 that Case 1 or Case 2 must always apply so that Case 3 exists only for the completeness of the construction.)

B. Let $p$ be the candidate of order $i$ and suppose $p$ has type $\alpha$. Cancel all 
candidates and potential conditions of order $>i$.

$$
\begin{gathered}
D^{s+1}=D^{s} \cup\left\{(p)_{-}\right\}, \quad d(\beta, s+1)= \begin{cases}d(\beta, s) & \text { if }(\beta \geq a \text { or } \beta \subseteq \alpha) \text { and lev } \beta \leq i, \\
-1 & \text { otherwise, }\end{cases} \\
E_{\beta}^{s+1}= \begin{cases}E_{\beta}^{s} \text { if }(\beta \geq \alpha \text { or } \beta \subseteq \alpha) \text { and } \operatorname{lev} \beta \leq i, \\
\varnothing \quad \text { otherwise, }\end{cases} \\
R_{j, \beta}^{s+1}= \begin{cases}R_{j, \beta}^{s} & \text { if }(\beta \geq \alpha \text { of } \beta \subseteq \alpha) \text { and lev } \beta \leq i, \\
\varnothing & \text { otherwise, }\end{cases} \\
A_{\beta}^{s+1}= \begin{cases}\left\{r \in A_{\beta}^{s}: r<p\right\} \cup\left\{r \in \bar{D}^{s+1}: r>d(s+1)\right\} \text { if } \beta=o, \\
\left\{r \in A_{\beta}^{s}: r<p\right\} & \text { if } \beta \neq o, \operatorname{lev} \leq i \text { and }(\beta \geq \alpha \text { or } \beta \subseteq \alpha), \\
\varnothing & \text { otherwise. }\end{cases}
\end{gathered}
$$

If $a=o$ do nothing more. If $a \neq o$ then appoint $(p, a, \sigma, k)$ to be the potential condition of order $i$ where $k$ is the least number such that $a_{k}=1$ and $\sigma$ is the constant one sequence of length $l$ lhp.

C. Let $r$ be the candidate of order $i$ that can be improved at stage $s+1$. Cancel all candidates and potential conditions of order $\geq i$. Choose the least $j, \beta, p$, and $q$ in that order satisfying the definition of improvement.

$$
\begin{aligned}
& D^{s+1}=D^{s} \text {, } \\
& d(\gamma, s+1)= \begin{cases}d(\gamma, s) \quad \text { if } \gamma>\beta * j \text { or } \gamma \subset \beta * j, \\
j\left(i, k\left(i,(q)_{-}, s\right), s\right) & \text { if } \gamma=\beta * j, \\
-1 \text { otherwise, } & \end{cases} \\
& E_{\gamma}^{s+1}=\left\{\begin{array}{l}
E_{\gamma}^{s} \quad \text { if } \gamma>\beta * j \text { or } \gamma \subset \beta * j, \\
E_{\gamma}^{s} \cup\{(p, q)\} \quad \text { if } \gamma=\beta * j, \\
\varnothing \text { otherwise, }
\end{array}\right. \\
& R_{k, \gamma}^{s+1}= \begin{cases}R_{k, \gamma}^{s} \quad \text { if } \gamma>\beta * j \text { or } \gamma \subset \beta * j, & \\
R_{k, \gamma}^{s} \cup\left[l\left(j,(p)_{-}, s\right), k\left(j,(p)_{\overrightarrow{1}}, s\right)\right] & \text { if } \gamma=\beta * j \text { and } k=0, \\
R_{k, \gamma}^{s} \cup\left[l\left(j,(q)_{\overline{0}}, s\right), k\left(j,(q)_{\overrightarrow{1}}, s\right)\right] & \text { if } \gamma=\beta * j \text { and } k=1, \\
\varnothing \quad \text { otherwise, } & \end{cases} \\
& A_{\gamma}^{s+1}= \begin{cases}\left\{a \in A_{\gamma}^{s}: a<p\right\} \cup\left\{a \in \bar{D}^{s+1}: a>d(s+1)\right\} \quad \text { if } \gamma=n, \\
\left\{a \in A^{s}: a<p\right\} & \text { if } \gamma>\beta * j \text { or }(\gamma \subset \beta * j \text { and } \gamma \neq 0), \\
A_{\gamma}^{s} \cup\{(p, q)\} & \text { if } \gamma=\beta * j, \\
\varnothing \quad \text { otherwise. } & \end{cases}
\end{aligned}
$$


If there exists a potential condition of order $i$ ' of the form $(a, \gamma, \sigma, k)$ where $i^{\prime}<i$ and $\gamma<\beta * j$ then cancel the candidate of order $i^{\prime}$ and hence the potential condition as well. (Note: we cannot have $\gamma \subseteq \beta * j$ otherwise $\beta * j$ would be frozen.) Appoint the point $(p, q)$ to be the candidate of order $i$.

D. Let $p$ be the least number of $\bar{A}_{o}^{s}$ such that $p>d(s), p>$ any candidate of order $<i$ and $p \geq m(i, o, s)$. Appoint $p$ to be the candidate of order $i$, cancel all candidates of order $>i$ and make no other changes at stage $s+1$ except to increase the arguments of functions to $s+1$.

This ends the construction. The set $D$ is r.e. since all the functions and predicates used in the construction are recursive.

We say that stage $s+1$ is an A-stage, B-stage, C-stage or D-stage pertaining to $i$ corresponding to the case that applies at stage $s+1$. We sometimes say that the candidate of order $i$ is the point considered at stage $s+1$.

Remarks on the construction.

1. If $\delta \neq \gamma$ then $E_{\delta}^{s} \cap E_{\gamma}^{s}=\varnothing$.

2. If $x \in E_{\delta}^{s}$ then ext $\delta=l b x$.

3. If $x<y, x \in E_{\delta}^{s}$, and $y \in E_{\gamma}^{s}$ then $\delta \geq \gamma$ or $\delta \subset \gamma$.

4. If $x \neq y, x \in A_{\delta}^{s}$, and $y \in A_{\gamma}^{s}$ then $x<y$ or $y<x$.

5. If $x \in A_{\delta}^{s}$ then $(x)_{\sigma} \in \bar{D}^{s}$ for all $\sigma$ such that $l b x=l b \sigma$.

6. If $x \in E_{\delta}^{s}$ and $l b \sigma=l b \tau=l b p$ then $\sigma<\tau$ if and only if $(x)_{\sigma}<(x)_{\tau}$.

7. $r(\delta, s) \leq d(\delta, s)$, and $d(\delta, s)=-1$ iff $r(\delta, s)=-1$.

8. If $x \in E_{\delta}^{s}$ and $\delta \neq o$ then $(x)_{1} \leq r(\delta, s)_{\text {. }}$

9. $R_{0, \delta}^{s} \cap R_{1, \delta}^{s}=\varnothing$.

10. If there exists a candidate of order $i$ and type $\delta$ in existence at stage $s$ then for each $\gamma \subset \delta$ there must have been a candidate of order $i$ and type $\gamma$ in existence at an earlier stage.

The proof of 1 through 10 is by induction on $s$. The remarks are clearly true for $s=0$. Assume the remarks are true for $s$. It is not difficult to check that the remarks are true if stage $s+1$ is an A-stage, a B-stage, or a D-stage. Let stage $s+1$ be a $C$-stage. Suppose stage $s+1$ pertains to $i$ and $j, \beta, p$, and $q$ are chosen. In most cases we need only consider the point $(p, q)$.

1. Let $\delta \neq \gamma$. If $\delta \neq \beta * j$ and $\gamma \neq \beta * j$ then $E_{\delta}^{s} \cap E_{\gamma}^{s}=\varnothing$ by the induction hypothesis. Let $\delta \neq \beta * j$. If $(p, q) \in E_{\delta}^{s+1}$ then $\delta>\beta * j$ or $\delta \subset \beta * j$ and $(p, q) \in E_{\delta^{*}}^{s}$. By the induction hypothesis $l b(p, q)=\operatorname{ext} \delta$. Thus $\delta \not \subset \beta * j$. If $\delta>\beta * j$ then $(p, q)<r(\delta, s)<l\left(j,(p)_{-}, s\right)<p$ by part (i) of the definition of improvement. This is impossible.

2. Since $j \geq \operatorname{lev} \beta$ then ext $\beta * j=\operatorname{ext} \beta+1$. Now, $p$ and $q$ are of type $\beta$ thus $l b p=l b q=\operatorname{ext} \beta$. Hence ext $\beta * j=l b(p, q)$.

3. First let $(p, q)<r$ and let $r$ be of type $\gamma$. If $\beta * j<\gamma$ then by part (i) of 
the definition of improvement $r<(p, q)$ which is impossible. Thus $\gamma \leq \beta * j$. On the other hand, let $r<(p, q)$ and let $r$ be of type $\delta$. If $\delta<\beta * j$ and $\delta \underline{\not} \beta * j$ then $E_{\delta}^{s+1}=\varnothing$.

4. Let $r \neq(p, q)$ and let $r \in A_{\delta}^{s+1}$. If $\delta=0$ then $r<(p, q)$ or $r>d(\beta * j, s+1) \geq((p, q))_{\overline{1}}$. If $\delta \neq 0$ and $\delta \neq \beta * j$ then by the construction $r \in A_{\delta}^{s}$ and $r<(p, q)$. If $\delta=\beta * j$ then by part (i) of the definition of improvement $r<(p, q)$.

5. Both $p$ and $q$ are members of $A_{\beta}^{s}$ and $(p, q) \in A_{\beta * j}^{s+1}$. By the induction hypothesis $((p, q))_{\sigma} \in \bar{D}^{s}$ for all $\sigma$ such that $l b(p, q)=l b \sigma$.

6. By part (b) of the definition of improvement $p<q$, hence the conclusion holds for $(p, q)$.

7. We must have $r(\beta * j, s+1)=k\left(j,(q)_{-}, s\right) \leq j\left(i, k\left(j,(q)_{\overline{1}}, s\right), s\right)=$ $d(\beta * j, s+1)$.

8. We have $(q)_{1} \leq k\left(j,(q)_{\overline{1}}, s\right)=r(\beta * j, s+1)$.

9. This is quite clear from (i) and (j) in the definition of improvement.

10. All we need show is that if $\gamma \subset \beta * j$ then there existed a candidate of order $i$ and type $\gamma$ at an earlier stage. At stage $s$ the candidate of order $i$ is of type $\alpha$ and $\beta \subseteq \alpha$ by part (d) of the definition of improvement. Hence $\gamma \subseteq \alpha$ and by the induction hypothesis there was a candidate of type $\gamma$ at an earlier stage.

IV. Candidates and potential conditions. In this section we prove three technical claims.

Claim 1. (i) If $i<j$ and $p$ and $q$ are candidates of orders $i$ and $j$ respectively both in existence at stage $s$ then $p<q$. (ii) If $p$ is the candidate of order $i$ and type $\alpha$ in existence at stage s then $p \in E_{\alpha}^{s}$. If $p$ is unimplemented then $p \in A_{\alpha}^{s}$ and if $p$ is implemented then there exists a potential condition of the form $(p, \alpha, \sigma, k)$ in existence at stage $s$.

The proof is by induction on $s$. Since no candidates are in existence at stage 0 then the claim holds for $s=0$. Assume the claim is true for $s$. We first prove part (i) of the claim for $s+1$. Since no new candidates are appointed in an A-stage or B-stage then we need only consider $s+1$ to be a C-stage or Dstage. Let $i, j, p$, and $q$ be given and let stage $s+1$ pertain to $k$. First suppose $s+1$ is a C-stage. We need only consider the case when $q$ is the new candidate of order $k$. By the induction hypothesis $p \in E_{\alpha}^{s}$ for some $\alpha$ and since $q$ was just chosen we have $q \in E_{\beta}^{s+1}$ for some $\beta$. If $p$ is unimplemented then by part ( $g$ ) of the definition of improvement $p<q$. If $p$ is implemented then by the induction hypothesis there exists a potential condition of the form $(p, \alpha, r, l)$ in existence at stage $s$. Now, $p$ is still the candidate of order $i$ at stage $s+1$ hence $\alpha \geq \beta$. 
For if $a<\beta$ then by the construction $p$ would have been cancelled as the candidate of order $i$ at stage $s+1$. By part (i) of the definition of improvement $p<q$. Now, suppose $s+1$ is a D-stage. We need only consider the case when $k=i$ and $p$ is appointed as the candidate of order $i$ at stage $s+1$. Stage $s+1$ cannot be the first stage when a candidate or order $i$ is appointed since a candidate of order $j$ exists at stage $s$. Let $u+1<s+1$ be the last stage when a candidate of order $i$ was cancelled. Any candidate of order $j$ was also cancelled at stage $u+1$ so that $q$ must have been appointed as a candidate after stage $u+1$ and before stage $s+1$. This is impossible.

We now proceed to prove part (ii) of the claim. Let $p$ be the candidate of order $i$ and type $\alpha$ in existence at stage $s+1$. Assume stage $s+1$ pertains to $j$. There are four cases to consider corresponding to the four types of stages possible.

Case A. If stage $s+1$ is in Case 1 or Case 3 there is nothing more to show. If stage $s+1$ is in Case 2 then $i \leq j$ otherwise $p$ is cancelled at stage $s+1$. By part (i) of the claim $p \leq q$ where $q$ is the candidate of order $j$ in existence at stage $s$. By the induction hypothesis $p \in E_{\alpha}^{s}$ and by Remark 3 and the construction $E_{a}^{s}=E_{a}^{s+1}$. Further, if $p$ is unimplemented then $p \in A_{a}^{s+1}$ since $p<q$. If $p$ is implemented then the potential condition associated with $p$ is either slightly changed at stage $s+1$ or persists unchanged since $p$ is not cancelled at stage $s+1$.

Case B. Again $i \leq j$ otherwise $p$ is cancelled. We have $p \leq q$ where $q$ is the candidate of order $j$ and type $\beta$. Now $E_{\alpha}^{s+1}=E_{\alpha}^{s}$ if $\alpha \subseteq \beta$ or $a \geq \beta$ and $\operatorname{lev} \alpha \leq j$. It is not difficult to see that lev $\alpha \leq i$ since $p$ is of type $\alpha$ and the level of the type of any point of order $i$ must be $\leq i$. Again by Remark $3, E_{a}^{s+1}=$ $E_{a}^{s}$. We now argue as in Case $A$.

Case C. If $i=j$ then the new candidate of order $i$ is a member of $A_{a}^{s+1}$ by definition. If $i<j$ then we argue as in Cases $\mathrm{A}$ and $\mathrm{B}$.

Case D. Trivial.

Claim 2. If $(p, a, \sigma, j)$ is the potential condition of order $i$ in existence at stage $s$ then

(i) $p$ is the candidate of order $i$ in existence at stage $s$ and is of type $a$,

(ii) $l h p=l b \sigma=\operatorname{ext} \alpha$,

(iii) $p$ is implemented, in fact, $(p)_{\tau} \in D^{s}$ for all $\tau \geq \sigma$ sucb that $l b \tau=l b \sigma$,

(iv) $a_{j}=1$,

(v) $j<i$,

(vi) $\sigma$ is not a constant zero sequence,

(vii) if $n=1+$ the number of zeros at the end of $\sigma$ then $j \leq n$th $k$ such that $a_{k}=1$.

The proof is quite straightforward by induction on $s$. Claim 2 verifies the 
parenthetical note that Case 3 of an A-stage never occurs.

Claim 3. Let $r$ and $r$ 'be unimplemented candidates of orders $i$ and $j$ and types $\alpha$ and $\beta$ respectively in existence at stage s. If $i<j$ and $\alpha \subseteq \beta$ then $\alpha_{k}=$ $\beta_{k}$ for all $k<i$. In particular, if $\operatorname{lev} \beta \leq i$ then $\alpha=\beta$.

The proof is by induction on $s$. The claim is vacuously true for $s=0$. Assume it is true for $s$. We need only consider a C-stage $s+1$ pertaining to $j$. Let $(p, q)$ be the new candidate of order $j$ and type $\gamma * l$. Let $r$ be the unimplemented candidate of order $i$ and type $\alpha$. Assume $a \subseteq \gamma * l$.

If $\alpha=\gamma * l$ then we are finished. So we can assume that $\alpha \subseteq \gamma$. We now show that $a_{k}=\gamma_{k}$ for all $k<i$. If $\gamma=o$ then there is nothing more to show. On the other hand if $\gamma \neq o$ let $s^{\prime}+1<s+1$ be the stage when $p$ came into existence. The point $r$ was already in existence at stage $s^{\prime}+1$ since $r<p$. Stage $s^{\prime}+1$ pertains to some $i^{\prime}>i$ otherwise $r$ would be cancelled as the candidate of order $i$ at stage $s^{\prime}+1$. Thus the candidate of order $i^{\prime}$ is $p$ of type $\gamma_{0}$. By the induction hypothesis, $a_{k}=\gamma_{k}$ for all $k<i$.

If $l \geq i$ then $a_{k}=(\gamma * l)_{k}$ for all $k<i$. On the other hand suppose $l<i$. We now show that the quadruple $l, \gamma, p, q$ also satisfied the definition of improvement of the candidate of order $i$ at stage $s+1$. The conditions (a), (b), (f), (h), (i), and $(j)$ hold since they are independent of $i$. We check the other conditions. (c) lev $\gamma \leq l<i$ since lev $\gamma * l \leq i$. Since $\gamma=\alpha$ then certainly $\gamma \subseteq a$ and $\gamma * l>a$. (e) $m(i, \gamma, s) \leq m(j, \gamma, s)<p$. (g) Since $p<$ any unimplemented candidate of order $\langle j$ then $p>$ any unimplemented candidate of order $<i$. Thus the condition of order $i$ requires attention at stage $s+1$ contrary to the hypothesis.

V. $D$ is nonrecursive. We begin by making the following claim.

Claim 4. For each i only finitely many stages pertain to $i$.

Assume the claim is true for all $i^{\prime}<i$. Let $t$ be the least stage after which no condition of order $<i$ requires attention. If there exists a potential condition of order $i$ at some stage $s$ then for all $s^{\prime} \geq s, D^{s^{\prime}} \cap w_{i}^{s^{\prime}} \neq \varnothing$. No candidate of order $i$ is chosen or improved after stage $s$. Once a potential condition of order $i$ is instituted no more than $i \cdot 2^{i}$ stages can pertain to $i$. Let us assume then that no potential condition of order $i$ ever comes into existence. If there exists no candidate of order $i$ in existence at stage $t+1$ then we must have $D^{t} \cap$ $W_{i}^{t} \neq \varnothing$. In this case the condition of order $i$ never requires attention after stage $t$. We can assume that there exists a candidate of order $i$ at stage $t+1$. At all stages $\geq t+1$ a candidate of order $i$ is in existence. Let $a$ be the lexicographically greatest member of $\Gamma$ such that there exists a stage $s>t$ and a candidate of order $i$ and type $\alpha$ in existence at stage $s$. There exists such an $\alpha$ since any 
candidate of order $i$ must have the level of its type $\leq i$ and there are only finitely many members of $\Gamma$ with level $\leq i$.

Let $s_{0}+1>t$ be the least stage pertaining to $i$ such that a candidate of order $i$ and type $\alpha$ is appointed at stage $s_{0}+1$. We now show that for all $s>s_{0}$ stage $s+1$ does not pertain to $i$. Suppose the contrary. Let $s_{1}+1$ be the least stage $>s_{0}+1$ pertaining to $i$. The unimplemented candidate of order $i$ in existence at stage $s_{0}+1$ is still in existence at stage $s_{1}+1$ since it cannot be cancelled by conditions of order $>i$. Stage $s_{1}+1$ must be a $C$-stage since no potential condition of order $i$ ever exists. The new candidate of order $i$ created at stage $s_{1}+1$ must have type $>a$. This is impossible.

Claim 5. $D$ is nonrecursive.

Let $i$ be any number. If $D \cap w_{i} \neq \varnothing$ then we are finished. If $D \cap w_{i}=\varnothing$ then let $t$ be a stage such that no stage $\geq t$ pertains to a condition of order $\leq i$. There must be a candidate $p$ of order $i$ in existence at stage $t$. Furthermore, for all $s \geq t, p$ remains in existence. Now, $(p)_{1}-W_{i}$ otherwise the condition of order $i$ would require attention at some stage $\geq t$. By Remark $5,(p)_{1} \in \bar{D}^{s}$ for all $s \geq t$. Thus $(p)_{\overline{1}} \in \bar{D} \cap \bar{W}_{i}$. Hence $\bar{D} \neq W_{i}$.

VI. Every r.e. set Turing equivalent to $D$ is mitotic. We make the following definitions.

$$
E_{a}=\bigcup_{s} \bigcap_{s^{\prime} \geq s} E_{a}^{s^{\prime}}, \quad A_{a}=\bigcup_{s} \bigcap_{s^{\prime} \geq s} A_{a}^{s^{\prime}}
$$

Claim 6. If $E_{a}$ and $E_{\beta}$ are both infinite then $a \subseteq \beta$ or $\beta \subseteq \alpha$.

Suppose $\alpha \leq \beta$. If $\alpha=0$ we are finished. If $\alpha \neq 0$ then for each $s$ if a new point is added to $E_{\beta}^{s}$ at stage $s+1$ we have $E_{a}^{s+1}=\varnothing$ unless $a \subseteq \beta$. Since $E_{\beta}$ in infinite then $E_{\alpha}^{s}=\varnothing$ for infinitely many $s$ unless $\alpha \subseteq \beta$.

Claim 6 justifies the following definition. Let $\alpha$ be the infinite sequence of 0 's and 1's such that $a_{i}=1$ if and only if there exists $a \in \Gamma$ such that $a_{i}=1$ and $E_{a}$ is infinite. If $a \in \Gamma$ then we can define $a \subseteq a$ just as we did earlier. It is not difficult to see that $\alpha \subseteq \alpha$ if and only if $E_{\alpha}$ is infinite. If $E_{\alpha}$ is infinite than $\alpha \subseteq \alpha$ by Claim 6. If $\alpha \subseteq \alpha$ then $\alpha \subseteq \beta$ where $E_{\beta}$ is infinite. But the points of $E_{\beta}$ are built up from the points of $E_{\alpha}$. Thus $E_{a}$ is infinite.

Claim 7. If $E_{\alpha}$ is infinite then $A_{\alpha}$ is infinite.

Since $E_{o}=N$ we first prove the claim for $a=o$. Let $i$ be any natural number. Let $t+1$ be the greatest stage which pertains to a condition of order $\leq i+1$ where $t+1$ is not an A-stage in Case 1 . Let $p$ be the $(i+1)$ st member 
of $A_{o}^{t+1}$. We show by induction on $s$ that for all $s \geq t+1$ any candidate of order $>i+1$ in existence at stage $s$ is $>p$ and, further, $p$ is the $(i+1)$ st member of $A_{o}^{s}$. Since stage $t+1$ is not an A-stage in Case 1 then all candidates and potential conditions of order $>i+1$ are cancelled at stage $t+1$. Let $s+1>t+1$. If stage $s+1$ is an $A$-stage in Case 1 then $A_{o}^{s}=A_{o}^{s+1}$. If stage $s+1$ is an $A$ stage in Case 2 or a B-stage then it must pertain to some condition of order $j>i+1$. By the induction hypothesis $p<$ the candidate of order $j$. Hence $p$ remains the $(i+1)$ st member of $A_{o}^{s+1}$. If stage $s+1$ is a C-stage or a D-stage then the new candidate of order $>i+1$ and is specifically chosen $\geq m(i+1, o, s)>p$. The point $p$ remains the $(i+1)$ st member of $A_{o}^{s+1}$. We conclude that $A_{o}$ contains at least $i+1$ members.

Let $a \neq o$ with level $m$ and let $n$ be any number. Assume $A_{a}$ has at least $n$ members. We show that $A_{a}$ has at least $n+1$ members. Let $t$ be a stage such that for all $s \geq t, E_{\alpha}^{s} \neq \varnothing$ and $A_{\alpha}^{s}$ contains the first $n$ members of $A_{\alpha \cdot}$. Choose $i \geq \max \{m, n+1\}$ such that $W_{i}=\varnothing$ and no stage $\leq t$ pertains to $i$. Such an $i$ exists since there exists infinitely many $i$ such that $W_{i}=\varnothing$. We now show that there exists a stage $s+1>t$ pertaining to $i$ such that the candidate of order $i$ is improved to type $a$. Since $E_{\alpha}$ is infinite there exists $i^{\prime}>i$ and $s^{\prime}+1>t$ such that the candidate of order $i^{\prime}$ is improved to type $\alpha$ at stage $s^{\prime}+1$. Let $\beta$ be the type of the candidate of order $i$ in existence at stage $s^{\prime}+1$. Such a candidate exists because $W_{i}=\varnothing$. By Claim 1 and Remark $3 \beta \geq \alpha$ or $\beta \subseteq \alpha$. If $\beta>\alpha$ and $\alpha \nsubseteq \beta$ then $E_{\alpha}^{s}=\varnothing$ for some $s \geq t$. If $\alpha \subseteq \beta$ then by Remark 10 at some stage prior to stage $s^{\prime}+1$ but after stage $t$ there must have been a candidate of order $i$ and type $\alpha$ in existence. If $\beta \subseteq \alpha$ then $\beta=\alpha$ by Claim 3 since lev $\alpha \leq i$.

Now, let $s_{0}+1$ be the last stage such that the candidate of order $i$ is improved to type $\alpha$. No stage $>s_{0}+1$ pertains to a condition of order $<i$ except if the stage is an A-stage in Case 1. If not then at some stage $s_{1}+1>s_{0}+1$ there is no candidate of order $i$ in existence at stage $s_{1}+1$. We can argue as we did in the last paragraph that at some stage $>s_{1}+1$ the candidate of order $i$ is improved to type $\alpha$ again. This is impossible. There are at least $n+1$ members in $A_{a}^{s_{0}+1}$. There are the $n$ members of $A_{a}^{s_{0}}$ plus the new candidate of order $i$ in $A_{a}^{s_{0}+1}$. Let $p$ be the $(n+1)$ st member of $A_{a}^{s_{0}+1}$.

We show by induction on $s$ if $s \geq s_{0}+1$ and $q$ is a candidate of order $>i$ in existence at stage $s$ then $q>p$, and further $p \in A_{a}^{s}$. This is clear for $s=s_{0}+1$ since there are no candidates of order $>i$ in existence at stage $s_{0}+1$. If stage $s+1$ is an $A$-stage in Case 1 then $A_{\alpha}^{s}=A_{\alpha}^{s+1}$. If stage $s+1$ is any other type of stage then it pertains to some $j \geq i$. If stage $s+1$ is an A-stage in Case 2 or B-stage then $j>i$ since $W_{i}=\varnothing$. By the induction hypothesis the candidate or order $j$ in existence at stage $s$ is $>p$ thus $p \in A_{a}^{s+1}$. If stage $s+1$ is a C-stage 
then let $\beta$ be the type of the new candidate of order $j$. If $\beta \leq a$ then clearly the new point of order $j$ is $>p$ and $p \in A_{\alpha}^{s+1}$. If $\alpha<\beta$ then $\alpha \subset \beta$ otherwise $E_{a}^{s+1}=\varnothing$ which is impossible. If $q$ is the new candidate of order $j$ then $m(j, \alpha, s)<q$. Now, $j \geq i \geq n+1$. Hence $q>$ the $(n+1)$ st member of $A_{\alpha}^{s}$ if there are at least $n+1$ members in $A_{a}^{s}$, which there are by the induction hypothesis. Thus $q>p$ and $p \in A_{a}^{s+1}$. If stage $s+1$ is a D-stage then $A_{a}^{s}=A_{a}^{s+1}$ and the candidate is chosen $>p$. We conclude that $A_{\alpha}$ contains at least $n+1$ members.

Claim 8. If $\theta_{j}(D)=Y_{j}$ and $\Psi_{j}\left(Y_{j}\right)=D$ then $a_{j}=1$.

Let $\beta$ be the greatest member of $\Gamma$ such that lev $\beta \leq j+1$ and $\beta \subseteq \alpha$. If $\beta_{j}=1$ we are finished. Assume for reductio ad absurdum that $\beta_{j}=0$. Clearly $E_{\beta *_{j}}$ must be finite.

We begin by showing that there exist at most finitely many stages $s$ such that a candidate of type $\geq \beta * j$ is appointed at stage $s$. Let $t$ be a stage such that for all $s \geq t, E_{\beta}^{s} \neq \varnothing$. If a candidate of type $\gamma \geq \beta * j$ is appointed at a stage $s \geq t$ then $\gamma \supset \beta$ otherwise $E_{\beta}^{s}=\varnothing$. If $p$ is appointed at stage $s$ as a candidate of type $\gamma$ where $\gamma \geq \beta * j$ and $\gamma \supset \beta$ then $p$ is built up from members of $E_{\beta * k}^{s}$ where $\operatorname{lev} \beta \leq k \leq j$. We can say that all types $\gamma$ such that $\gamma \geq \beta * j$ and $\gamma \supset \beta$ are built up from finitely many types of the form $\beta * k$ where $\operatorname{lev} \beta \leq k \leq j$. So it suffices to show that for all $k$ such that $\operatorname{lev} \beta \leq k \leq j$ there exist at most finitely many stages $s$ such that a candidate of type $\beta * k$ is appointed at stage $s$. Suppose not. Let $k$ be the least number $\geq \operatorname{lev} \beta$ such that there exist infinitely many stages $s$ such that a candidate of type $\beta * k$ is appointed at stage $s$. Let $t_{0}$ be a stage $\geq t$ such that no candidate of type $\gamma$ is appointed at any stage $\geq t_{0}$ where $\gamma \supseteq \beta * l$ and $\operatorname{lev} \beta \leq l<k$. Choose $t_{1} \geq t_{0}$ such that if $s+1 \geq t_{1}$ then at stage $s+1$ no candidate of type $\gamma$ is considered where $\gamma \supseteq \beta * l$ and $\operatorname{lev} \beta \leq l<k$ and further $s+1$ does not pertain to a number $\leq k+1$. We now argue that if $s \geq t_{1}$ then $E_{\beta * k}^{s} \subseteq E_{\beta * k}^{s+1}$. Now, by the construction $E_{\beta * k}^{s+1}=\varnothing$ or $E_{\beta * k}^{s} \subseteq E_{\beta * k}^{s+1}$. If $E_{\beta * k}^{s+1}=\varnothing$ and $E_{\beta * k}^{s} \neq \varnothing$ then either stage $s+1$ considers a candidate of type $\gamma>\beta * k$ (as in Parts A (Case 2), B, and C of the construction) or some candidate of order $\leq k+1$ is implemented in part $\mathrm{B}$ of the construction. Since the second of these two possibilities is impossible then $\gamma \supseteq \beta$ or else $E_{\beta}^{s+1}=\varnothing$. Hence $\gamma \supseteq \beta$ and $\gamma>\beta * k$. This implies there exists an $l$ such that $\operatorname{lev} \beta \leq l<k$ such that $\gamma \supseteq \beta * l$ which is also impossible. Hence $E_{\beta * k}^{s} \subseteq E_{\beta * k}^{s+1}$ for all $s \geq t_{1}$. We conclude that $E_{\beta * k}$ is infinite since infinitely many candidates are appointed which are of type $\beta * k$. Now, lev $\beta * k \leq j+1$ since $k \leq j$. This contradicts the assumption that $\beta$ is the greatest member of $\Gamma$ such that $\operatorname{lev} \beta \leq j+1$ and $E_{\beta}$ is infinite.

Let $s_{0}$ be a stage such that, for all $s \geq s_{0}, E_{\beta}^{s} \neq \varnothing$ and no candidate of 
type $\geq \beta * j$ is appointed at stage $s$. Let $i$ be the least number such that no candidate of order $i$ has been chosen by stage $s_{0}, j<i$ and $w_{i}=\varnothing$. Let $s_{1} \geq s_{0}$ be a stage such that no stage $\geq s_{1}$ pertains to a condition of order $\leq i$. At stage $s_{1}$ there exists a candidate $r$ of order $i$ and type $\alpha$ for some $\alpha$. Clearly $r$ is the candidate of order $i$ for all $s \geq s_{1}$. Since $E_{\beta}$ is infinite there exists $i^{\prime}>i$ such that at some stage $s \geq s_{1}$ a candidate of order $i^{\prime}$ and type $\beta$ is appointed. Since $E_{a}^{s} \neq \varnothing$ then $\alpha>\beta$ or $\alpha \subseteq \beta$.

If $\alpha>\beta$ then $\alpha \supset \beta$ otherwise $E_{\beta}^{s^{\prime}}=\varnothing$, where $s^{\prime} \geq s_{0}$ is the stage when $r$ was chosen as the candidate of order $i$. If $\alpha \subseteq \beta$ then $\alpha=\beta$ by Claim 3. In any case $\beta \subseteq \alpha$. Since $s_{1} \geq s_{0}$ then $\beta * j>\alpha$.

Since $E_{\beta}$ is infinite so is $A_{\beta}$. Choose points $p$ and $q$ as follows.

(i) $p \in A_{\beta}$ and $q \in A_{\beta}$,

(ii) $p<q$,

(iii) $p>$ any candidate ever appointed of order $<i$,

(iv) $p>\max \{m(i, \beta, s): s \in N\}$,

(v) $\lim _{s} l\left(j,(p)_{-}, s\right)>\max \{r(\gamma, s): \gamma \geq \beta * j$ and $s \in N\}$,

(vi) $\lim _{s} k\left(j,(p)_{-}, s\right)<\lim _{s} l\left(j,(q)_{0}, s\right)$.

Such $p$ and $q$ exist because $\theta_{j}(D)=Y_{j}$ and $\Psi_{j}\left(Y_{j}\right)=D$ and there exist only finitely many stages when a candidate of type $\geq \beta * j$ is appointed.

Let $s_{2} \geq s_{1}$ be a stage such that

(1) $p$ and $q$ are in existence at all stages $\geq s_{2}$,

(2) $\beta$ is not frozen at stage $s_{2}$,

(3) $L(j, s)>\lim _{s} k\left(j,(q)_{1}, s\right)$ for all $s \geq s_{2}$,

(4) no stage $\geq s_{2}$ considers a candidate of type $\geq \beta * j$,

(5) no candidate of type $\geq \beta * j$ is cancelled at any stage $\geq s_{2}$.

Since $p$ and $q$ are in $A_{\beta}^{s}$ for all $s \geq s_{2}$ it must be the case that $l\left(j,(p)_{0}, s_{2}\right)=$ $\lim _{s} l\left(j,(p)_{-}, s\right), l\left(j,(q)_{-}, s_{2}\right)=\lim _{s} l\left(j_{,}(q)_{0}, s\right)$ and $k\left(j,(p)_{-}, s_{2}\right)=$ $\lim _{s} k\left(j,(p)_{1}, s\right)$. Clearly the candidate of order $i$ can be improved at stage $s_{2}+1$ if we can show that $\beta * j$ itself is not frozen at stage $s_{2}$. If $\beta * j$ is frozen at stage $s_{2}$ then there exists at stage $s_{2}$ a potential condition of the form $(a, \gamma$, $\sigma, k)$ where if $\delta$ is the greatest member of $\Gamma$ such that $\delta \subseteq \gamma$ and $\delta \subseteq \beta * j$, and $\sigma=\sigma^{\prime} * \sigma^{\prime \prime}$ where $l b \sigma^{\prime \prime}=\operatorname{ext} \delta$ then $\sigma^{\prime \prime}$ has a nonzero member. Now, $\delta \underline{\beta} \beta$ since otherwise $\beta$ is frozen at stage $s_{2}$. We conclude that $\delta=\beta * j$. Thus $\gamma \supseteq \beta * j$ and so $\gamma \geq \beta * j$. This potential condition is never cancelled for if it were then (5) above fails. If $k<j$ then the potential condition is eventually satisfied since $\beta_{k}=1$ implies $\lambda s L(k, s)$ is unbounded. Thus $k \geq j$ otherwise (4) fails. Further $k \neq j$ since $\lambda s L(j, s)$ is unbounded by the hypothesis of the claim. Hence $k>j$. Let $n=1+$ the number of zeros at the end of $\sigma$. By Claim $2, k \leq n$th $l$ 
such that $\gamma_{l}=1$. Now, $\sigma=\sigma^{\prime} * \sigma^{\prime \prime}$ where $l b \sigma^{\prime \prime}=\operatorname{ext} \beta * j$ and $\sigma^{\prime \prime}$ contains a nonzero member. Hence $n \leq \operatorname{ext} \beta * j$. Since $k<n$th $l$ such that $\gamma_{l}=1$ then $k$ must be $\leq j$. We cannot have both $k>j$ and $k \leq j$ so we cannot have $\beta * j$ frozen at stage $s_{2}$.

We have shown that the condition of order $i$ requires attention at stage $s_{2}+1 \geq s_{0}$ which is impossible. Our assumption that $\beta_{j}=0$ is false.

Claim 9. If $\theta_{j}(D)=Y_{j}$ and $\Psi_{j}\left(Y_{j}\right)=D$ then there exists r.e. sets $Y_{j, 0}$ and $Y_{j, 1}$ such that $Y_{j, 0} \cap Y_{j, 1}=\varnothing, Y_{j, 0} \cup Y_{j, 1}=Y_{j}, D \leq_{T} Y_{j, 0}$, and $D \leq_{T}^{j, 0} Y_{j, 1}$.

Let $a \subseteq a$ be such that $\operatorname{lev} a=j+1$. Such an $a$ exists by Claim 8. Let $t_{0}$ be the last stage such that $E_{\alpha}^{t_{0}}=\varnothing$. For all $s \geq t_{0}, E_{\alpha}^{s} \subseteq E_{\alpha}^{s+1}, R_{0, \alpha}^{s} \subseteq R_{0, \alpha}^{s+1}$, $R_{1, \alpha}^{s} \subseteq R_{1, \alpha}^{s+1}$ and $d(\alpha, s) \leq d(\alpha, s+1)$. Let $R=\bigcup\left\{R_{0, a}^{s}: s \geq t_{0}\right\}$. The set $R$ is recursive since the intervals that make up $R$ are enumerated in increasing order. Define $Y_{j, 0}^{s}=Y_{j}^{s} \cap R$ and $Y_{j, 1}^{s}=Y_{j}^{s} \cap \bar{R}$. Now define $Y_{j, 0}=U_{s} Y_{j, 0}^{s}$ and $Y_{j, 1}=$ $\bigcup_{s} Y_{j, 1}^{s}$. Clearly $Y_{j, 0} \cap Y_{j, 1}=\varnothing$ and $Y_{j, 0} \cup Y_{j, 1}=Y_{j} \cdot$

We now show that $D \leq_{T} Y_{j, 1}$. The proof that $D \leq_{T} Y_{j, 0}$ is analogous. Let $M=\max \{d(\beta, s): \beta>\alpha, \beta \underline{\not} \alpha$ and $s \in N\}$. M exists otherwise $E_{a}=\varnothing$. We now show how to compute $D(x)$ for $x>M$ inductively from $Y_{j, 1}$.

Let $x>M$. Compute the least number $t_{1} \geq t_{0}$ such that $d\left(a, t_{1}+1\right) \geq x$. The stage $t_{1}$ exists because $E_{a}$ is infinite. Let $t_{2}$ be the least stage $\geq t_{1}+1$ such that

(i) $D(y)=D^{2} 2(y)$ for all $y<x$,

(ii) $Y_{1, j}(y)=Y_{1, j}^{t_{2}}(y)$ for all $y \leq r\left(a, t_{1}+1\right)$,

(iii) $L\left(j, t_{2}\right)>r\left(a, t_{1}+1\right)$,

(iv) $a$ is not frozen at stage $t_{2}$.

We will show that $x \in D$ iff $x \in D^{t}$.

For reductio ad absurdum let us suppose that $x \in D-D^{t}$. Suppose $x \in D^{s} 2^{+1}-D^{s}$. Let $p$ be the member of $E_{a}$ created at stage $t_{1}+1$.

Let $\rho$ be the constant zero sequence of length ext $\alpha-1$. We begin by making the following.

Subclaim. There exists a candidate $q$ of type $\beta \supseteq \alpha$ in existence at stage $s_{2}$ such that $x=(p)_{0 * \rho}$ and $p=(q)_{\sigma}$ for some $\sigma$ of length $\operatorname{ext} \beta-\operatorname{ext} \alpha$.

Assume that the subclaim has been proved and let $\beta, q$, and $\sigma$ be as in the subclaim. Let $r$ be the constant one sequence of length ext $\alpha-1$. The number $(p)_{1 * \tau}$ must enter $D$ at some stage $s_{0}+1<s_{2}+1$. At stage $s_{0}+1$ a potential condition of the form $(q, \beta, \sigma * 1 * r, l)$ comes into existence. Furthermore at every stage $s$ such that $s_{0}+1 \leq s \leq s_{2}$ a potential condition of the form 
$(q, \beta, \sigma * 1 * v, l)$ where $l b v=\operatorname{ext} \alpha-1$ is in existence otherwise $x=(p)_{0 * p}$ would never enter $D$ at stage $s_{2}+1$. Hence $\alpha$ is frozen at each stage $s$ such that $s_{0}+1 \leq s \leq s_{2}$. Since $t_{2} \leq s_{2}$ then $t_{2} \leq s_{0}$ otherwise $a$ would be frozen at stage $t_{2}$. Let $s_{1}+1$ be the stage such that $(p)_{1 * \rho} \in D^{s_{1}+1}-D^{s_{1}}$. Now, $s_{0}<$ $s_{1}<s_{2}$. At stage $s_{1}+1$ a potential condition of the form $(q, \beta, \sigma * 1 * \rho, l)$ where $l=$ the least number such that $\beta_{l}=1$ comes into existence. There are exactly ext $\alpha-1$ zeros at the end of $\sigma * 1 * \rho$. Hence the number $n$ referred to in Case 1 of part $A$ of the construction is actually equal to ext $\alpha$. Eventually a potential condition of the form $(q, \beta, \sigma * 1 * \rho, j)$ must come into existence since $j=$ the $(\operatorname{ext} \alpha)$ th $k$ such that $\alpha_{k}=1$. Let $t_{3}$ be the stage when this potential condition is satisfied and $(p)_{0 * \tau}$ actually enters $D$ at stage $t_{3}+1$. We show now that $j,(p)_{1 * \rho},(p)_{1 * r}, t_{1}, t_{2}, t_{3}$ satisfy the basic lemma. We certainly have $(p)_{1 * \rho} \leq(p)_{1 * \tau}$ and $t_{1} \leq t_{2}<t_{3}$. Now, the point $\left((p)_{0},(p)_{1}\right)$ was constructed at stage $t_{1}+1$. Hence $L\left(j, t_{1}\right)>k\left(j,(p)_{1 * r}, t_{1}\right)$ and $d\left(\alpha, t_{1}+1\right)=$ $j\left(j, k\left(j,(p)_{1 * \tau}, t_{1}\right), t_{1}\right)$. Since $(p)_{1 * \tau}$ enters $D$ at stage $s_{0}+1$ and $s_{0} \geq t_{2}$ then no number $\leq d\left(\alpha, t_{1}+1\right)$ may enter $D$ at a stage $s$ such that $t_{1} \leq s \leq t_{2}$. Furthermore, no number $<(p)_{1 * \rho}$ may enter $D$ at a stage $s$ such that $t_{1} \leq s \leq t_{3}$ otherwise $(p)_{0 * \tau}$ would never enter $D$ at stage $t_{3}+1$. Now, $L\left(j, t_{2}\right)>r\left(\alpha, t_{1}+1\right)=$ $k\left(j,(p)_{1 * r}, t_{1}\right)$ by the definition of $t_{2}$ and $L\left(j, t_{3}\right)>(p)_{1 * \rho}$ since the potential condition $(q, \beta, \sigma * 1 * \rho, j)$ is satisfied at stage $t_{3}$. All the hypotheses of the basic lemma are satisfied. Hence there exists a $z \in Y_{j}^{t^{3}}-Y_{j}^{t^{2}}$ such that $l\left(j,(p)_{1 * \rho}, t_{1}\right)<z \leq k\left(j,(p)_{1 * \tau}, t_{1}\right)$. Now, $\left[l\left(j,(p)_{1 * \rho}, t_{1}\right), k\left(j,(p)_{1 * \tau}, t_{1}\right)\right] \subseteq \bar{R}$. Hence $z \in Y_{j, 1}^{t_{3}}-Y_{j, 1}^{t_{2}}$ and $z \leq r\left(\alpha, t_{1}+1\right)$. This contradicts part (ii) of the definition of $t_{2}$. We conclude that our supposition that $x \in D-D^{t_{2}}$ is false.

We now proceed to prove the subclaim. There certainly exists a candidate $q$ of some type $\beta$ in existence at stage $s_{2}$ such that $x=(q)_{v}$ for some $v$ of length equal to ext $\beta$. We must have $\beta \leq \alpha$ or $\alpha \subseteq \beta$ since otherwise $E_{a}^{s} 2^{+1}=\varnothing$. If $\beta \leq \alpha$ then we can conclude that $\beta \subseteq \alpha$ otherwise $p<q$ which is impossible since $x \leq(p)_{1}$. Assume $\beta \subseteq \alpha$. We shall show that $\beta=\alpha$. Now, $\alpha$ is frozen from the stage when $(q)_{\overline{1}}$ enters $D$ until at least stage $s_{2}$. Hence $(q)_{\overline{1}}$ enters $D$ after stage $t_{2}$. Now, $q$ is the candidate of order $i$ for some $i$. We cannot have $i \leq j$ or else $E_{a}^{s+1}=\varnothing$ where $s+1>t_{2}\left(s+1\right.$ is the stage when $(q)_{1}$ enters $\left.D\right)$. Let $u_{1}+1$ be the stage when $q$ was chosen as the candidate of order $i$. The point $p$ was chosen at stage $t_{1}+1$ to be the candidate of order $i^{\prime}$ for some $i^{\prime}$. We must have $u_{1} \leq t_{1}$ or else $q>p$ which is impossible. Hence at stage $t_{1}+1$ both $p$ and $q$ are unimplemented candidates of orders $i^{\prime}$ and $i$ respectively. Now $i \leq i^{\prime}$ or else $q>p$. Now, lev $\alpha=j+1 \leq i \leq i$ ' hence by Claim $3 \beta=\alpha$.

So far we have shown that $a \subseteq \beta$. Since $x=(q)_{\nu}$ then $x=\left((q)_{\sigma}\right)_{\mu}$ for some $\sigma$ 
of lenth ext $\beta-\operatorname{ext} \alpha$ and $\mu$ of length ext $\alpha$. Since $t_{1}+1$ is the least stage such that $x \leq d\left(\alpha, t_{1}+1\right)$ and $p$ was created at stage $t_{1}+1$ then $(q)_{\sigma}$ must be $p$. It remains to show that $\mu$ is the constant zero sequence of length ext $\alpha$. At stage $s_{2}+1$ a potential condition of order $i$ of the form $(q, \beta, \sigma * \mu, k)$ is appointed where $k$ is the least number such that $\beta_{k}=1$. Suppose that $\mu$ is not the constant zero sequence of length ext $\alpha$. Then $\alpha$ is frozen at stage $s_{2}+1$. Further $\alpha$ must be frozen at every stage $s \geq s_{2}+1$ via a potential condition of order $i$ of the form $(q, \beta, \sigma * \mu, l)$ for some $l$. This is shown by induction on $s$. There is no problem if $s+1$ is an A-stage in Case 1 or a D-stage. There are three cases left to consider.

(1) $s+1$ is an A-stage in Case 2. Let $i^{\prime}$ be the order of the potential condition satisfied at stage $s+1$. If $i^{\prime} \leq i$ then some number $<x$ must enter $D$ at stage $s+1$ which is impossible. If $i<i^{\prime}$ then the potential condition of order $i$ remains in force.

(2) $s+1$ is a B-stage. Let $i$ be the order of the candidate implemented at stage $s+1$. Again we must have $i<i^{\prime}$ or else some number $<x$ enters $D$ at stage $s+1$. On the other hand if $i<i^{\prime}$ then the potential condition remains in force.

(3) $s+1$ is a C-stage. The new candidate $r$ must be of order $i^{\prime}$ and of type $\gamma$ where $\gamma \leq \alpha$ or $\gamma \supseteq a$ since otherwise $E_{a}^{s+1}=\varnothing$. Since $\alpha$ is frozen at stage $s$ then we cannot have $\gamma \supseteq a$. Since $\gamma \leq \alpha$ and $a \subseteq \beta$ then $\gamma \leq \beta$. We of course cannot have $i=i^{\prime}$. If $i<i^{\prime}$ then $(q, \beta, \sigma * \mu, l)$ could only be cancelled if $\gamma>\beta$ which is not the case. Suppose $i^{\prime}<i$. Let $a$ be the candidate of order $i^{\prime}$ in existence at stage $s$ just before $r$ was chosen and suppose $a$ has type $\delta$. Now, since $i^{\prime}<i$ then $a<q$ and $\delta \geq \beta$ or $\delta \subseteq \beta$. Since $\gamma$ is to be an improvement over $\delta$ and $\gamma \leq \beta$ then $\delta \leq \beta$. Hence $\delta \subseteq \beta$. Let $u$ be the stage when $q$ was chosen as the candidate of order $i$. The point $a$ must be the candidate of order $i$ in existence at stage $u$. By Claim $3 \delta_{k}=\beta_{k}$ for all $k<i^{\prime}$. Since $\gamma>\delta$ and lev $\gamma \leq i^{\prime}$ then there exists $k^{\prime}<i^{\prime}$ such that $\gamma_{k}=\delta_{k}$ for all $k<k^{\prime}, \gamma_{k^{\prime}}=1$ and $\delta_{k}{ }^{\prime}=0$. Hence we must have $\gamma>\beta$ which is impossible. We conclude that $\mu$ is the constant zero sequence of length equal to ext $\alpha$.

This concludes the proof of the subclaim. The theorem is now proved where $\mathrm{d}$ is the degree of $D$.

VII. Proofs of the corollaries. Recall that we need to prove Corollaries 1 and 3 and the fact that $d^{\prime \prime}=0^{\prime \prime}$.

Proof of Corollary 1. The fact that every r.e. set of degree $d$ is strongly mitotic is proved simply by noticing in the proof of Claim 9 that $R$ is recursive, $Y_{j, 0} \subseteq R$, and $Y_{j, 1} \subseteq \bar{R}$.

Proof of Corollary 3. To show that every two r.e. sets of degree $d$ are weak 
truth table equivalent we show that every r.e. set of degree $d$ is weak truth table equivalent to $D$. Let $\theta_{j}(D)=Y_{j}$ and $\Psi_{j}\left(Y_{j}\right)=D$. The reduction procedure used in Claim 9 to reduce $D$ to $Y_{j, 1}$ is a weak truth table reduction procedure since the number $d\left(t_{1}+1\right)$ can be found effectively from $x$. Hence $D \leq_{\mathrm{w}} Y_{j, 1}$ (" $\leq_{\mathrm{w}}$ " means "weak truth table in"). Trivially, $Y_{j, 1} \leq_{w} Y_{j}$. Therefore $D \leq_{w} Y_{j}$. To show that $Y_{j} \leq_{\mathrm{w}} D$ let $t_{0}$ be as in Claim 9 and let $y$ be any number. Let $u_{1}$ be the least number $\geq t_{0}$ such that $r\left(a, u_{1}+1\right) \geq y$. Let $u_{2}$ be the least stage $\geq u_{1}+1$ such that

(i) $D(x)=D^{u} 2(x)$ for all $x \leq d\left(\alpha, u_{1}+1\right)$,

(ii) $d\left(\alpha, u_{2}+1\right)>d\left(\alpha, u_{2}\right)$.

If we can show that $y \in Y_{j}$ iff $y \in Y_{j}^{u}{ }^{2}$ then we have exhibited a reduction procedure that witnesses $Y_{j} \leq{ }_{w} D$.

Let $p$ be the candidate of type $a$ created at stage $u_{2}+1$. We first show that no number $<(p)_{0}$ may enter $D$ after stage $u_{2}$. Suppose not, let $x<(p)_{\delta}$ be the least number that enters $D$ after stage $u_{2}$. We must have $x>d\left(a, u_{1}+1\right)$ hence $x=(q)_{\sigma}$ for some $\sigma$ and $q \in E_{a}$. Let $u+1$ be the stage when $q$ was created. Clearly $u_{2}+1 \geq u+1>u_{1}$ and $d(\alpha, u+1)>d(\alpha, u)$. Furthermore, we must have $D(z)=D^{u}(z)$ for all $z \leq(q)_{0}$ since $x$ is the least number that enters $D$ after stage $u_{2}$ and no number $<(q)_{0}$ may enter $D$ between stage $u+1$ and $u_{2}$ (or else $x$ never would have been put into $D)$. S Since $q>d\left(\alpha, u_{1}+1\right)$ then $D(z)=D^{u}(z)$ for all $z \leq d\left(\alpha, u_{1}+1\right)$. We conclude that $u+1=u_{2}+1$ and $p=q$.

Since no number $<(p)_{0}$ may enter $D$ after stage $u_{2}$ then using an argument like that in the basic lemma no number $\leq l\left(j,(p)_{\overline{0}}, u_{2}\right)$ may enter $Y_{j}$ after stage $u_{2}$. For if $z \in Y_{j}-Y_{j}^{u^{2}}$ and $z \leq l\left(j,(p)_{0}, u_{2}\right)$ then $Y_{j}(z) \neq \theta_{j}{ }^{2}\left(D^{u}{ }^{j} ; z\right)$. The computation on the right-hand side is protected since $D(w)=D^{u}(w)$ for all $w<(p)_{0}$. Now we specifically chose $r\left(a, u_{1}+1\right)<l\left(j,(p)_{\delta}, u_{2}\right)$. Hence $Y_{j}(z)=$ $Y_{j}^{u}{ }^{2}(z)$ for all $z \leq r\left(\alpha, u_{1}+1\right)$ and in particular $Y_{j}(y)=Y_{j}^{u}{ }^{2}(y)$ which was to be proved.

Proof that $\mathrm{d}^{\prime \prime}=0^{\prime \prime}$ [due to Paul F. Cohen]. First notice that if $A$ is r.e. and $A \leq_{T} D$ then $A \leq_{\mathrm{w}} D$. For if $A \leq_{T} D$ then $A \oplus D \equiv_{T} D$. Hence, by Corollary 3, $A \oplus D \leq_{\mathrm{w}} D$. Trivially then $A \leq_{\mathrm{w}} D$. From this we conclude that $\left\{e: W_{e} \leq_{T} \mathrm{~d}\right\}=$ $\left\{e: W_{e} \leq_{w} D\right\}$. Now $\left\{e: W_{e} \leq_{w} D\right\} \in \Sigma_{3}$ since the set is defined by the predicate

\section{$\exists i \exists j \forall x \forall s \exists s^{\prime}$}

$$
\begin{aligned}
& {\left[s^{\prime} \geq s, \phi_{i}^{s^{\prime}}(x)\right. \text { is defined, }} \\
& \left.\quad \Phi_{j}^{s^{\prime}}\left(D^{s^{\prime}}, x\right) \text { is defined and equal to } W_{e}^{s^{\prime}}(x) \text { and } c\left(j, D^{s^{\prime}}, x, s^{\prime}\right) \leq \phi_{i}^{s^{\prime}}(x)\right]
\end{aligned}
$$

Now, $\left\{e: W_{e} \leq_{T} \mathrm{~d}\right\} \in \Sigma_{3}$ and further $\mathrm{d}<0^{\prime}$ since $0^{\prime}$ contains a nonmitotic r.e. 
set. By a theorem of Yales $\left[6\right.$, Theorem 9] $\mathrm{d}^{\prime \prime}=0^{\prime \prime}$. This proof parallels a proof of Jockusch [1, Corollary 8].

VIII. Conclusion and open questions. Let $P(W)$ be a property of r.e. sets satisfying the following conditions:

(1) if $P(W)$ then $W$ is nonrecursive,

(2) there exists a $W$ such that $P(W)$ and $W \in 0^{\prime}$,

(3) for all nonrecursive r.e. $V$ there exists $W \leq_{T} V$ such that $P(W)$,

(4) there exists a nonrecursive r.e. $V$ such that for no $W \equiv_{T} V$ is $P(W)$ true.

The properties " $W$ is nonmitotic" and " $W$ is nonautoreducible" satisfies (1)-(4). From Sasso [5, Theorem 4] and from Corollary 3 we see that the property "there exists a r.e. $V$ such that $W \equiv_{T} V$ and $W \equiv_{\mathrm{w}} V$ " also satisfied (1)-(4). Are there any other interesting properties of r.e. sets that satisfy (1)-(4)? In particular, can a lattice theoretic property (lattice of r.e. sets) satisfy (1)-(4)?

There are several questions concerning the restrictions on the degrees of nonmitotic r.e. sets.

(a) Does the degree $d$ in the present construction have jump $=0^{\prime}$ ? If not then can the construction be modified to produce such a degree?

(b) Does $b$ r.e. and $b^{\prime}=0^{\prime \prime}$ or $b^{\prime \prime}>0^{\prime \prime}$ imply that $b$ contains a nonmitotic r.e. set?

(c) Are the degrees of the nonmitotic r.e. sets dense in the r.e. degrees?

The following list contains conjectures that we feel may be solvable using modifications of the techniques of this paper.

(i) There exists a nonrecursive r.e. degree containing only one r.e. weak truth table degree and also having a nonmitotic r.e. set.

(ii) There exists a completely mitotic nonrecursive r.e. degree containing more than one r.e. weak truth table degree.

(iii) There exists a completely mitotic nonrecursive r.e. degree which is not completely strongly mitotic.

Acknowledgments. We are indebted to A. H. Lachlan for his invaluable help during the preparation of this paper. Among other things he suggested a form of the basic lemma and recognized that the construction would be an "e-state" argument. We would also like to thank R. W. Robinson and C. G. Jockusch for reading several versions of this paper and providing many useful suggestions. We are grateful to L. P. Sasso for several enlightening conversations concerning weak truth table reducibility which led to our realization of Corollary 3 . We are grateful to Paul F. Cohen for improving our realization that $d^{\prime}<0^{\prime \prime}$ to $d^{\prime \prime}=0^{\prime \prime}$. This research was supported in part by NRC grant A-3040 and NSF grant GJ-34745X.

\section{BIBLIOGRAPHY}

1. C. G. Jockusch, Jr., Degrees in which the recursive sets are uniformly recursive, Canad. J. Math. (to appear). 
2. R. E. Ladner, Mitotic recursively enumerable sets, J. Symbolic Logic 38 (1973).

3. H. Rogers, Jr., Theory of recursive functions and effective computability, McGraw-Hill, New York, 1967. MR 37 \#61.

4. L. P. Sasso, Jro, Deficiency sets and bounded information reducibilities, Trans. Amer. Math. Soc. (to appear).

5. C. E. M. Yates, Three theorems on the degrees of recursively enumerable sets, Duke Math. J. 32 (1965), 461-468. MR 31 \#4721.

6. - On the degrees of index sets. II, Trans. Amer. Math. Soc. 135 (1969), 249-266. MR 39 \#2637.

DEPARTMENT OF MATHEMATICS, UNIVERSITY OF CALIFORNIA, BERKELEY, CALIFORNIA 97420

DEPARTMENT OF MATHEMATICS, SIMON FRASER UNIVERSITY, BURNABY, BRITISH COLUMBIA, CANADA

COMPUTER SCIENCE GROUP, UNIVERSITY OF WASHINGTON, SEATTLE, WASHINGTON 98195 (Current address) 